\title{
Monoamine oxidase A mediates prostate tumorigenesis and cancer metastasis
}

\author{
Jason Boyang Wu, ${ }^{1}$ Chen Shao, ${ }^{1,2}$ Xiangyan Li, ${ }^{1}$ Qinlong Li, ${ }^{1,3}$ Peizhen Hu, ${ }^{1,3}$ Changhong Shi, ${ }^{1}$ \\ Yang Li, ${ }^{4}$ Yi-Ting Chen, ${ }^{1}$ Fei Yin, ${ }^{4}$ Chun-Peng Liao, ${ }^{1,5}$ Bangyan L. Stiles, ${ }^{4}$ Haiyen E. Zhau, ${ }^{1}$ \\ Jean C. Shih, ${ }^{4,6}$ and Leland W.K. Chung 1

\begin{abstract}
1Uro-Oncology Research Program, Department of Medicine, Cedars-Sinai Medical Center, Los Angeles, California, USA. ${ }^{2}$ Department of Urology and ${ }^{3}$ Department of Pathology, Xijing Hospital, Fourth Military Medical University, Xi'an, Shaanxi, China. ${ }^{4}$ Department of Pharmacology and Pharmaceutical Sciences, School of Pharmacy, and ${ }^{5}$ Center for Applied Molecular Medicine, Department of Medicine, Keck School of Medicine, University of Southern California, Los Angeles, California, USA. 6 USC-Taiwan Center for Translational Research,
\end{abstract} University of Southern California, Los Angeles, California, USA.

\begin{abstract}
Tumors from patients with high-grade aggressive prostate cancer (PCa) exhibit increased expression of monoamine oxidase A (MAOA), a mitochondrial enzyme that degrades monoamine neurotransmitters and dietary amines. Despite the association between MAOA and aggressive $\mathrm{PCa}$, it is unclear how MAOA promotes PCa progression. Here, we found that MAOA functions to induce epithelial-to-mesenchymal transition (EMT) and stabilize the transcription factor HIF1 $\alpha$, which mediates hypoxia through an elevation of ROS, thus enhancing growth, invasiveness, and metastasis of PCa cells. Knockdown and overexpression of MAOA in human PCa cell lines indicated that MAOA induces EMT through activation of VEGF and its coreceptor neuropilin-1. MAOA-dependent activation of neuropilin-1 promoted AKT/FOXO1/TWIST1 signaling, allowing FOXO1 binding at the TWIST1 promoter. Importantly, the MAOA-dependent HIF1 $\alpha /$ VEGF-A/FOXO1/ TWIST1 pathway was activated in high-grade PCa specimens, and knockdown of MAOA reduced or even eliminated prostate tumor growth and metastasis in PCa xenograft mouse models. Pharmacological inhibition of MAOA activity also reduced PCa xenograft growth in mice. Moreover, high MAOA expression in PCa tissues correlated with worse clinical outcomes in PCa patients. These findings collectively characterize the contribution of MAOA in PCa pathogenesis and suggest that MAOA has potential as a therapeutic target in PCa.
\end{abstract}

\section{Introduction}

Prostate cancer $(\mathrm{PCa})$ is the second leading cause of male cancer death in the Western world (1). It can be clinically categorized into different risk groups primarily based on histological grade (Gleason score), clinical TNM stage, and levels of serum prostatespecific antigen (2). Aggressive, poorly differentiated high-grade $\mathrm{PCa}$ is incurable and potentially lethal, underscoring the need for a greater understanding of the molecular basis of PCa progression and improved opportunities to eliminate the development of the lethal phenotype of PCa.

Monoamine oxidase A (MAOA) is a mitochondria-bound enzyme that catalyzes the degradation of monoamine neurotransmitters and dietary amines by oxidative deamination, which produces a by-product, hydrogen peroxide, a major source of ROS (3-5). ROS can predispose cancer cells to DNA damage and cause tumor initiation and progression (6). In the last several decades, MAOA has been widely studied in the context of neuropsychiatric disorders, such as aggressive behaviors and mental depression $(3,5)$. Recently, a significant correlation was established between increased levels of MAOA expression and high Gleason grade or poorly differentiated human prostate tumors $(7,8)$. MAOA is exclusively expressed in the epithelial cells of prostatic glands with relatively low levels in stromal counterparts (9). These observations collectively suggest that MAOA may function in an autocrine manner to regulate the proliferation and differentiation of prostatic epithelial cells.

Conflict of interest: The authors have declared that no conflict of interest exists. Citation for this article: J Clin Invest. 2014;124(7):2891-2908. doi:10.1172/JCI70982.
Prostate tumorigenesis and cancer development are regulated by several oncogenic cues leading to dysregulated growth and increased stemness and plasticity by which cancer cells acquire increased migratory, invasive, and metastatic potential through epithelial-to-mesenchymal transition (EMT) (10). Evidence also supports the ability of cancer cells to adapt a HIF $1 \alpha$ pathway to resist oxidative stress, which cooperatively promotes an increasingly aggressive phenotype in cancer cells $(11,12)$.

In this study, we proposed that increased MAOA expression in high-grade PCa may be an important contributor to its dysregulated growth and dedifferentiation of the glandular epithelial phenotype. We demonstrated the ability of MAOA to induce mesenchymal transition, with PCa cells gaining increased proliferative, invasive, and metastatic potentials. Moreover, genetic targeting of MAOA using shRNA effectively inhibited or even completely eliminated prostate tumorigenesis and cancer metastasis in mouse xenograft models. We showed mechanistically that MAOA potentiated aggressive PCa behavior by converging functional interplay among EMT, hypoxia, and oxidative stress. Additionally, evidence for MAOA functionality in PCa also extended to human clinical PCa specimens. These findings establish MAOA as a viable therapeutic target in $\mathrm{PCa}$ and provide a rationale for the development of MAOA-targeted therapeutics.

\section{Results}

MAOA suppresses epithelial phenotype and promotes mesenchymal transition. Although MAOA expression has been previously demonstrated in human PCa tissues (7), its mode of action and potential to drive aggressive PCa phenotypes such as increased EMT-medi- 
A
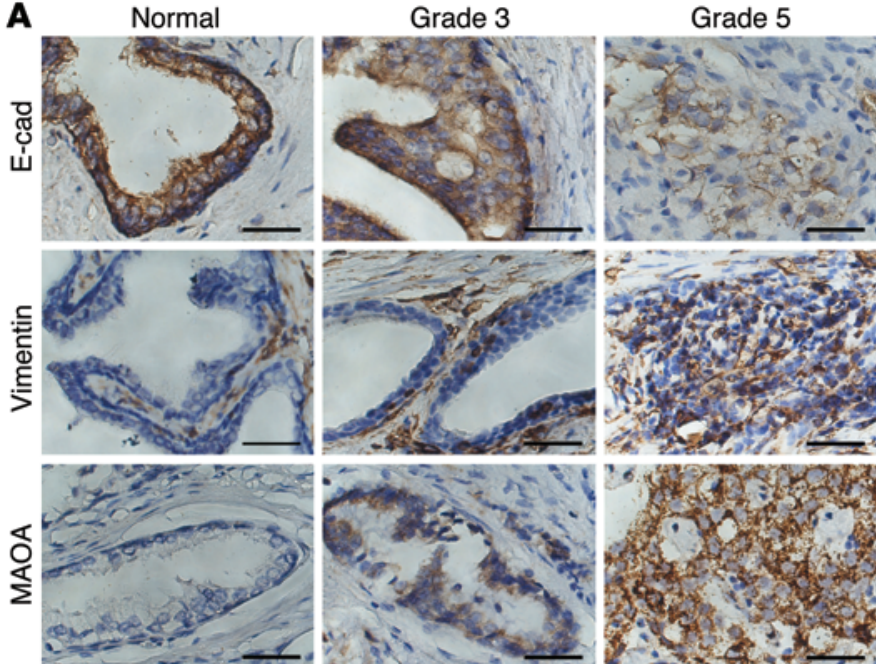

B
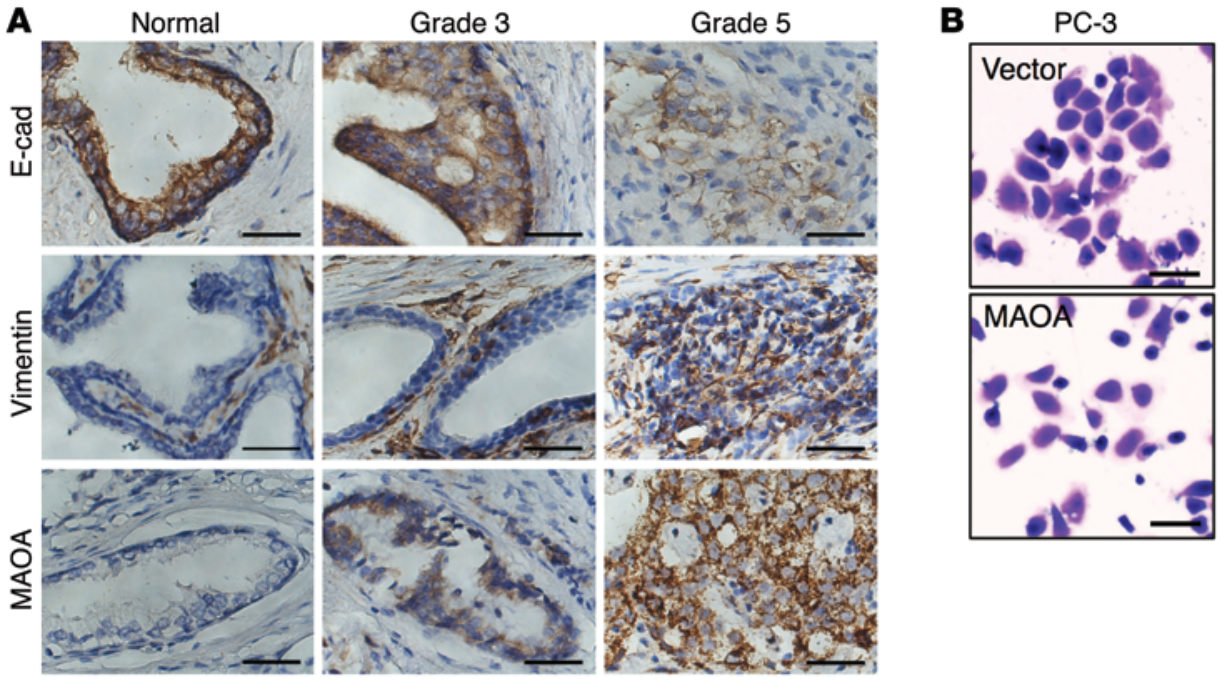

\section{c}

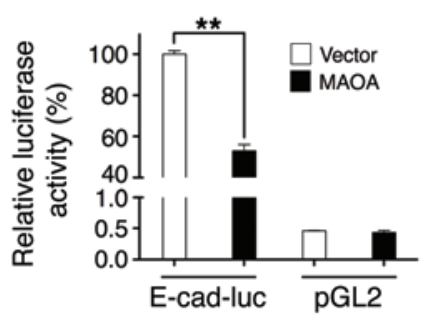

E

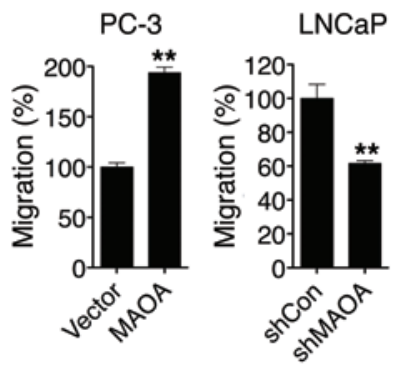

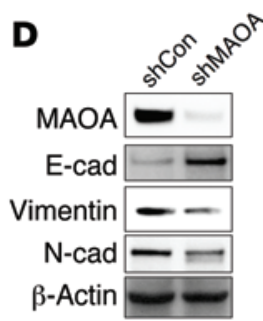

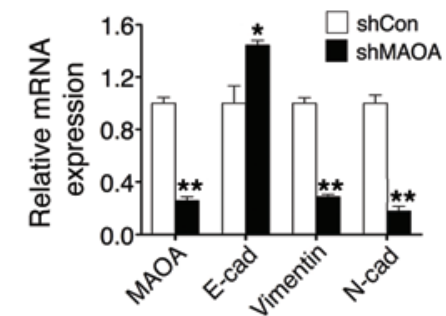

$\mathbf{F}$
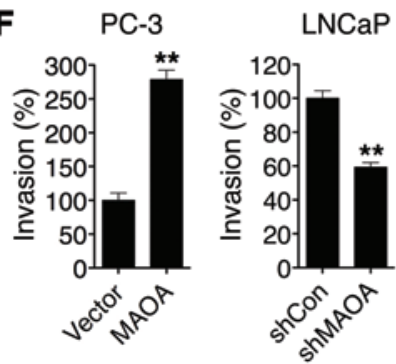

Figure 1

MAOA and EMT in PCa. (A) Clinical specimens of normal prostatic epithelium and Gleason grade 3 and 5 PCa were stained for E-cadherin, vimentin, and MAOA. Representative images from a tissue microarray are shown. Original magnification, $\times 400$; scale bars: $20 \mu \mathrm{m}$. (B) PC-3 cells stably overexpressing an empty vector or MAOA were photographed after crystal violet staining (left), and extracts were analyzed for the expression of MAOA and EMT markers by immunoblotting (middle) and qPCR (right). Original magnification, $x 40$; scale bars: $200 \mu \mathrm{m}$. ${ }^{\star} P<0.05$, ${ }^{* \star} P<0.01$. (C) Stable vector- and MAOA-overexpressing PC-3 cells were transfected with either an E-cadherin promoter reporter construct (E-cad-luc, expressing Firefly luciferase) or a promoterless pGL2 vector (expressing Firefly luciferase), cotransfected with a pRL-TK (expressing Renilla luciferase) construct as an internal standard for normalization of transfection efficiency. Data represent the mean \pm SEM $(n=3)$ of Firefly luciferase activity normalized to Renilla luciferase activity. The E-cadherin promoter activity in vector-expressing PC-3 cells was set as $100 \%$. ${ }^{* \star} P<0.01$. (D) Immunoblotting (left) and qPCR (right) analysis of LNCaP cells that express a MAOA-targeting shRNA (shMAOA) or a scrambled shRNA (shCon) for the expression of MAOA and EMT markers. ${ }^{\star} P<0.05,{ }^{\star \star} P<0.01$. (E and F) Paired PC-3 and LNCaP cells as indicated were assayed for their ability to either migrate $(\mathbf{E})$ or invade $(\mathbf{F})$. The migration or invasion of respective control cells was set as $100 \%$. Data represent the mean $\pm \operatorname{SEM}(n=3) .{ }^{*} P<0.01$.

ated signaling pathways have not been pursued. Using a series of clinical specimens as the gold standards, we observed consistently that high-Gleason grade (grade 5) PCa is distinguished from lowgrade (grade 3) PCa by characteristic morphological features such as the merger of neoplastic glands and cytological dedifferentiation (13). High-Gleason grade tumors also expressed diminished levels of E-cadherin (an epithelial marker) and increased expression of vimentin (a mesenchymal marker) and MAOA in the same clinical specimens (Figure 1A), which was further confirmed in a tissue microarray by quantification of the association of expression levels of these genes in different types of prostate tissues (Supplemental Figure 1, A and B; supplemental material available online with 
A

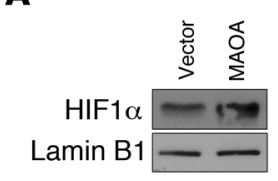

B

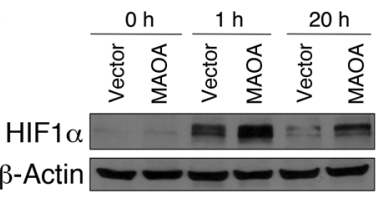

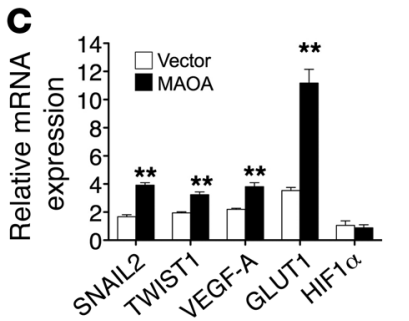

D

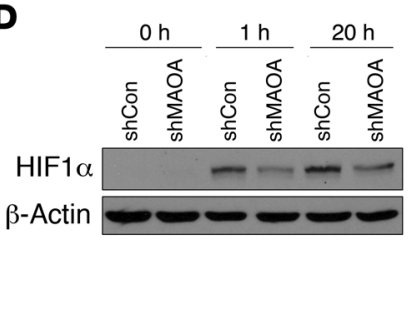

Figure 2

MAOA regulates HIF1 $\alpha$ stability. (A) Immunoblots of nuclear extracts from PC-3 (vector and MAOAoverexpression) cells cultured at $21 \% \mathrm{O}_{2}$ for HIF1 $\alpha$. (B and D) Immunoblots of paired PC-3 (B) or LNCaP (D) cells cultured at $1 \% \mathrm{O}_{2}$ for the indicated time. (C and E) Fold induction of HIF1 $\alpha$ target genes in response to hypoxia $\left(1 \% \mathrm{O}_{2}, 24\right.$ hours) measured by qPCR in PC-3 (vector and MAOA-overexpression) (C) or LNCaP (shCon and shMAOA) (E) cells. Normoxic gene expression in control PC-3 (vector-expressing) or LNCaP (shCon) cells was used as a control for normalization. Data represent the mean \pm SEM $(n=3) .{ }^{*} P<0.05,{ }^{* *} P<0.01$.

this article; doi:10.1172/JCI70982DS1). These results suggest that high-Gleason grade cancers exhibit EMT characteristics associated with increased MAOA expression and aggressive behaviors, which led us to hypothesize that MAOA may regulate EMT in PCa.

To address this hypothesis, we used human PC-3 and LNCaP PCa cell lines as models, since these cell lines express either low (PC-3) or high (LNCaP) basal levels of MAOA (Supplemental Figure 2, A-C). Stably enforced expression of a MAOA expression construct in PC-3 cells resulted in the transition to a dispersed morphology, a significant loss of E-cadherin, and increased expression of vimentin, $\mathrm{N}$-cadherin, and TWIST1 at both protein and mRNA levels (Figure 1B). To establish that MAOA regulates E-cadherin transcription, we assayed the activity of E-cadherin promoter-luciferase reporter gene in PC-3 cells that stably expressed either an empty vector or a MAOA construct. MAOA-overexpressing cells displayed substantially diminished promoter activity compared with control cells (Figure 1C). By contrast, stable knockdown of MAOA in LNCaP cells with a MAOA-targeting shRNA (shMAOA) increased the expression of E-cadherin and downregulated vimentin and $\mathrm{N}$-cadherin at both protein and mRNA levels compared with control cells given a scramble shRNA (shCon) (Figure 1D). Moreover, overexpression of MAOA also led to a significant increase in migration and invasion of PC-3 cells, a characteristic function of EMT. In contrast, knockdown of MAOA in LNCaP cells reduced the ability of cells to migrate or invade (Figure 1, E and F). In these studies, we did not observe significant changes in either cell proliferation or cell death in parallel to the analysis of cell migration and invasion in these cells with the manipulation of MAOA expression. Stable knockdown of MAOA in 2 other human PCa cell lines, C4-2 and $\mathrm{ARCaP}_{\mathrm{M}}$, supported the concept that MAOA is responsible for driving EMT in PCa cells, in which markedly attenuated mesenchymal features with changes in cell morphology, reduced mesenchymal marker expression, and reduced cell migratory and invasive behaviors were observed (Supplemental Figure 3, A-C).

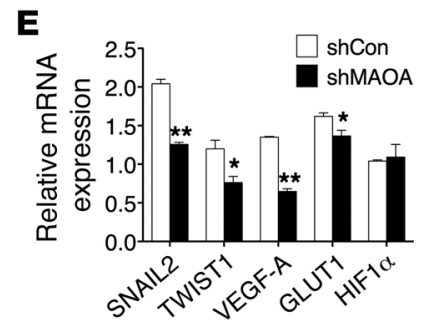

MAOA stabilizes HIF1 $\alpha$ via ROS generation and PHD destruction. Hypoxia, a common condition found in a wide range of solid tumors including PCa, is often associated with poor prognosis and frequent expression of an aggressive phenotype promoted by EMT. HIF $1 \alpha$, a master mediator of hypoxia, mediates hypoxic effects by activating relevant downstream target genes involved in many aspects of tumor progression, such as increased tumor glycolysis, angiogenesis, invasion, migration, and metastasis (14). Since increased MAOA expression promotes EMT, we assessed a possible relationship between MAOA and HIF $1 \alpha$.

This possibility was evaluated first by investigation of whether MAOA directly regulates HIF $1 \alpha$ stability under normoxic conditions. In the presence of physiological levels of oxygen, HIF $1 \alpha$ is rapidly degraded, which prevents a direct measurement in whole-cell lysates, but HIF $1 \alpha$ is detectable from isolated nuclei (15). As shown in Figure 2A, nuclei isolated from MAOA-overexpressing PC-3 cells during normoxia revealed elevated levels of HIF $1 \alpha$ relative to the vector-expressing control cells. Likewise, when these cells were cultured under hypoxic conditions at $1 \% \mathrm{O}_{2}, \mathrm{HIF} 1 \alpha$ was stabilized earlier and to a higher degree in MAOA-overexpressing cells when compared with control cells in whole-cell lysates (Figure 2B). Consistently, PC-3 tumors grown as subcutaneous xenografts from MAOA-overexpressing cells demonstrated increased staining of HIF1 $\alpha$ protein compared with control tumors by immunohistochemical (IHC) analysis (Supplemental Figure 4D). Moreover, select HIF1 $\alpha$ target genes, including the EMT-promoting genes SNAIL2 and TWIST1, VEGFA, and glucose transporter 1 (GLUT1), were significantly upregulated in MAOA-overexpressing cells compared with control cells under hypoxia. Enforced expression of MAOA and hypoxia further showed additive effects on the expression of these genes (Figure 2C). In contrast, stable knockdown of MAOA reduced HIF $1 \alpha$ stabilization as well as expression of its target genes (SNAIL2, TWIST1, VEGF-A, and GLUT1) in hypoxia-treated LNCaP cells, indicating that MAOA is required for HIF $1 \alpha$ function (Figure 2, D and E). Notably, the HIF1A mRNA level was not affected by differential MAOA expression (Figure 2, C and E), suggesting that MAOA may regulate HIF1 $\alpha$ stability via translational or posttranslational modifications. We assessed further the potential involvement of HIF $1 \alpha$ in MAOA-induced EMT and cell behaviors by genetically knocking down HIF1 $\alpha$ in hypoxiatreated MAOA-overexpressing PC-3 cells. We found that HIF1 $\alpha$ knockdown attenuated MAOA-induced EMT characteristics by decreasing mesenchymal marker expression and cell migration, invasion, and proliferation (Supplemental Figure 5, A-C). These data in aggregate suggest that MAOA modulates the stabilization of HIF $1 \alpha$ protein and the induction of crucial HIF $1 \alpha$ target genes that coordinate EMT and cancer progression. 
A

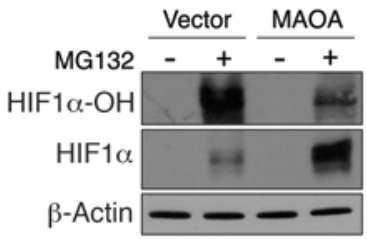

B

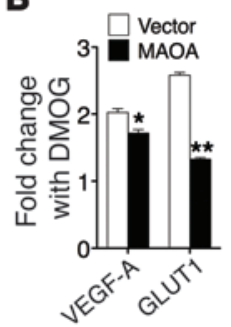

C

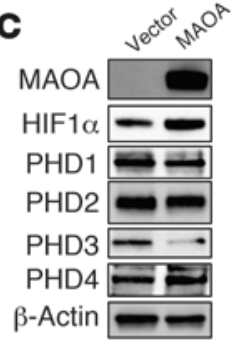

D

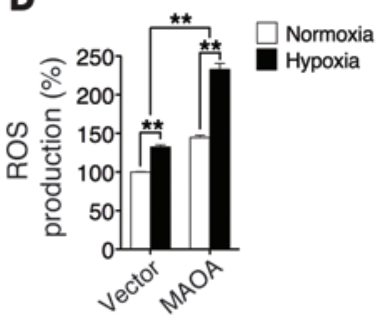

E

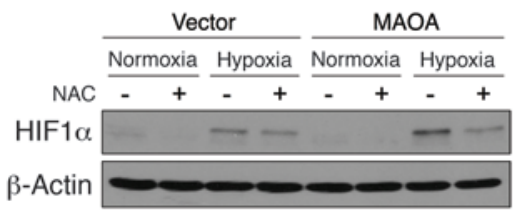

$\mathbf{F}$

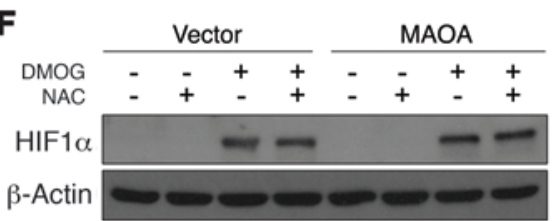

G

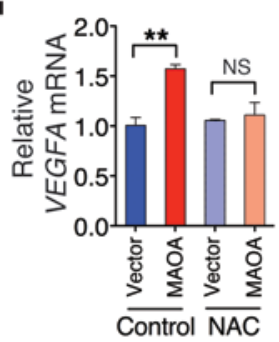

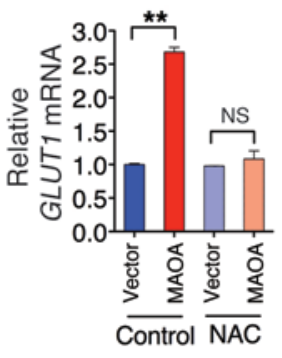

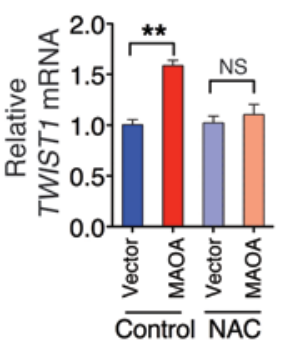

H

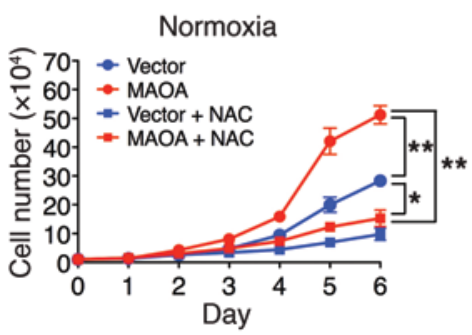

Hypoxia

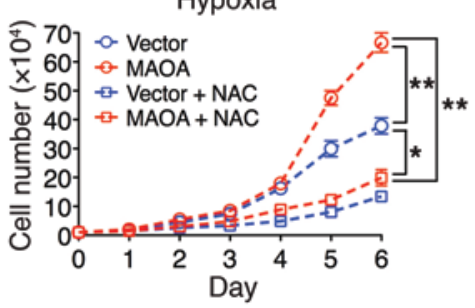

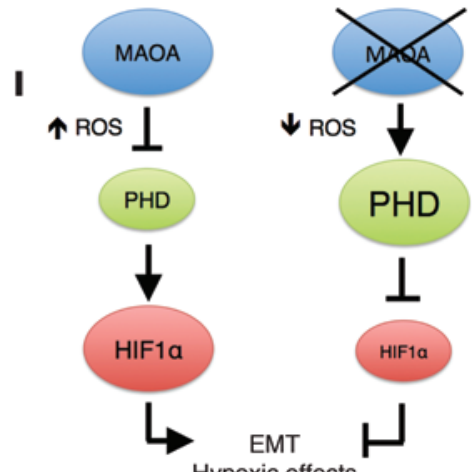

Hypoxic effects

Increased tumorigenesis

\section{Figure 3}

MAOA regulates HIF1 $\alpha$ stability through ROS. (A) Immunoblots of PC-3 (vector and MAOA-overexpression) cells treated with or without MG132 (1 $\mu \mathrm{M}, 6$ hours) for hydroxylated HIF1 $\alpha(\mathrm{HIF} 1 \alpha-\mathrm{OH})$ and total HIF1 $\alpha$. (B) Fold induction of HIF1 $\alpha$ target genes in PC-3 (vector and MAOA-overexpression) cells treated with DMOG (1 mM, 24 hours) was measured by qPCR, and the ratio (mean $\pm \mathrm{SEM}, n=3)$ of DMOGtreated to untreated gene expression is shown. ${ }^{*} P<0.05$, ${ }^{* *} P<0.01$. (C) Immunoblots of $P C-3$ (vector and MAOA-overexpression) cells with hypoxia for PHD1-4. (D) The increase in ROS production in PC-3 (vector and MAOA-overexpression) cells with hypoxia was calculated as the percentage changes (mean $\pm \mathrm{SEM}, n=3$ ) in ROS levels in hypoxic cells relative to normoxic cells. ${ }^{*} P<0.01$. (E) Immunoblots of PC-3 (vector and MAOA-overexpression) cells incubated with $10 \mathrm{mM} \mathrm{NAC}$ and cultured under normoxia and hypoxia. (F) Immunoblots of PC-3 (vector and MAOA-overexpression) cells cultured at $21 \% \mathrm{O}_{2}$ with $10 \mathrm{mM} \mathrm{NAC}$ or $1 \mathrm{mM} \mathrm{DMOG}$ as indicated. (G) qPCR analysis of VEGFA, GLUT1, and TWIST1 expression (mean \pm SEM, $n=3$ ) in PC-3 (vector and MAOA-overexpression) cells incubated with 10 mM NAC and cultured under hypoxia. ${ }^{* \star} P<0.01$. (H) Growth curves of PC-3 (vector and MAOA-overexpression) cells cultured in standard media supplemented or not supplemented with $10 \mathrm{mM}$ NAC under either normoxia (left panel) or hypoxia (right panel) (mean $\pm \mathrm{SEM}, n=3$ ). ${ }^{*} P<0.05$, ${ }^{\star \star} P<0.01$. (I) A schematic diagram outlining MAOA stabilization of HIF1 $\alpha$ by repression of PHD activity through ROS production.

The regulation of HIF $1 \alpha$ is complex and has not been fully elucidated. During normoxia, HIF $1 \alpha$ is hydroxylated at 2 proline residues by a family of oxygen-dependent prolyl hydroxylases (PHD1-4), which enables the tumor suppressor von Hippel-Lindau (vHL) to bind to and target HIF1 $\alpha$ for ubiquitination and protea- somal degradation (16). Since the HIF1A mRNA level remained unchanged by MAOA expression (Figure 2, C and E), we tested whether MAOA exerted a posttranslational effect on HIF1 $\alpha$ protein stability. We ruled out the direct interaction between these 2 proteins because of their distinct cellular localization. MAOA appears 
A

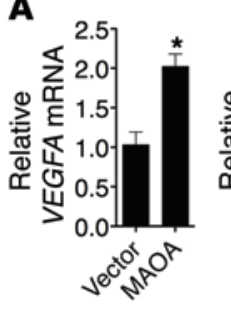

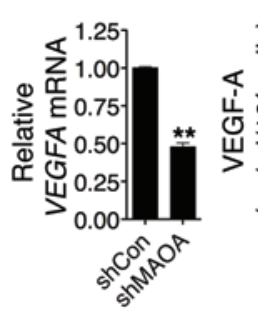

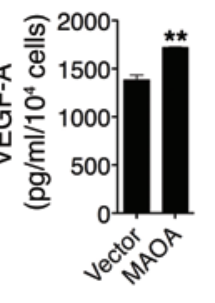

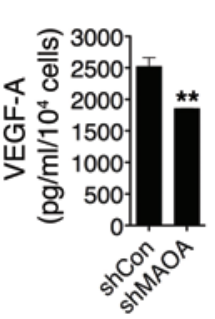

B
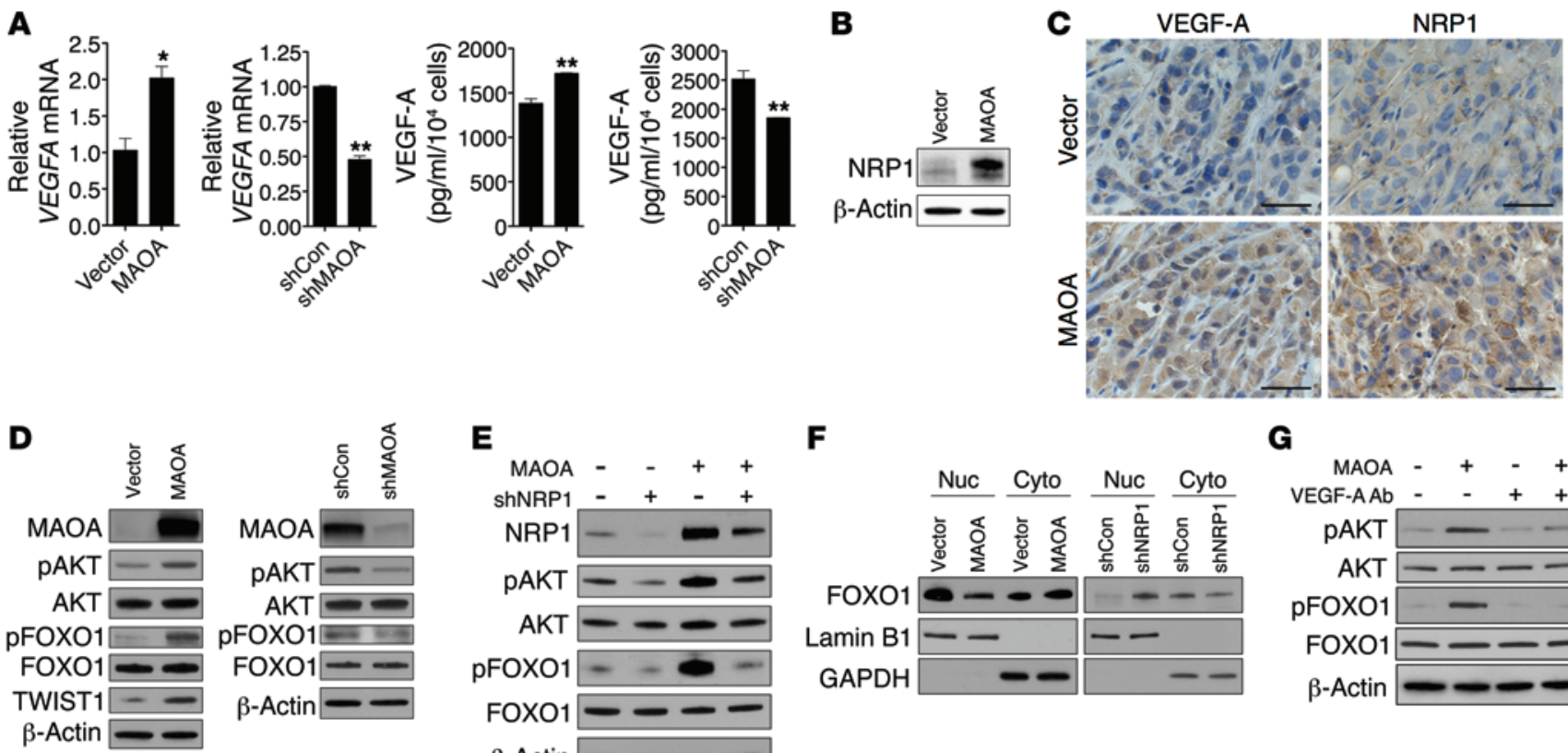

E

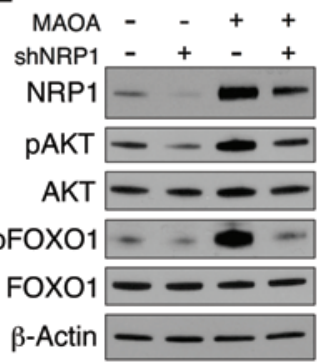

$\mathbf{F}$

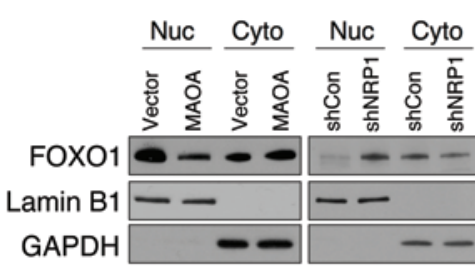

G

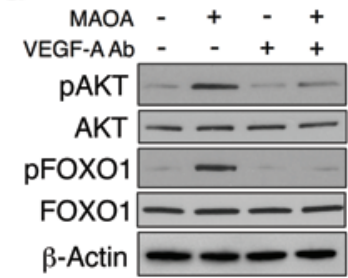

H

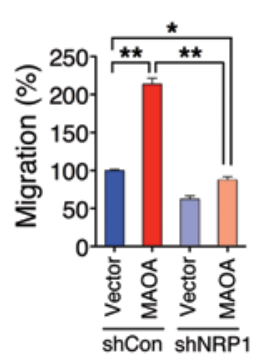

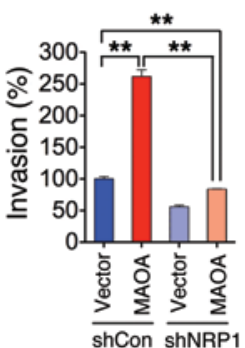

I

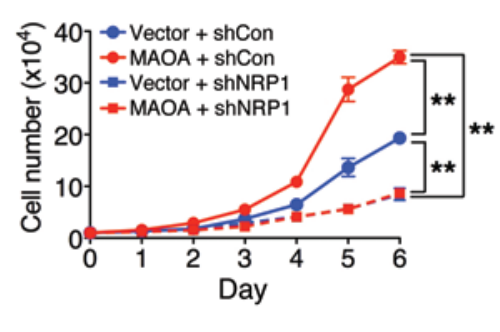

Figure 4

MAOA promotes VEGF-A/NRP1-mediated activation of AKT/FOXO1 signaling. (A) qPCR analysis of VEGFA mRNA expression (mean $\pm \mathrm{SEM}$, $n=3$ ) in paired PC-3 and LNCaP cells as indicated (left). VEGF-A secretion (mean \pm SEM, $n=3$ ) in culture media from these cells was quantified by ELISA (right). ${ }^{*} P<0.05,{ }^{*} P<0.01$. (B) Immunoblots of PC-3 (vector and MAOA-overexpression) cells for NRP1. (C) IHC analysis of PC-3 (vector and MAOA-overexpression) tumor xenografts for VEGF-A and NRP1 expression. Representative images from 5 separate samples are shown. Original magnification, $\times 400$; scale bars: $20 \mu \mathrm{m}$. (D) Immunoblots of paired PC-3 and LNCaP cells as indicated for PAKT (Ser473), pFOXO1 (Thr24), total AKT, and FOXO1. (E) Immunoblots of PC-3 (vector and MAOA-overexpression) cells that express NRP1-targeting shRNAs (shNRP1) or a scrambled shRNA (shCon) for NRP1, pAKT (Ser473), and pFOXO1 (Thr24). (F) Immunoblots of nuclear and cytoplasmic extracts from different groups of paired PC-3 cells as indicated for FOXO1. Lamin B1 and GAPDH serve as nuclear and cytoplasmic protein markers, respectively. (G) Immunoblots of PC-3 (vector and MAOA-overexpression) cells treated with either anti-VEGF-A antibody or a control IgG $(0.5 \mathrm{\mu g} / \mathrm{ml}, 24$ hours) for pAKT (Ser473) and pFOXO1 (Thr24). (H) PC-3 cells as established in E were assessed for their ability to either migrate (left) or invade (right). Data represent the mean $\pm \mathrm{SEM}(n=3) .{ }^{*} P<0.05,{ }^{* \star} P<0.01$. (I) Growth curves of PC-3 cells as established in E. Data represent the mean \pm SEM $(n=3) .{ }^{* *} P<0.01$.

in the outer membrane of mitochondria to execute enzymatic reactions (17) (Supplemental Figure 6, A-C), whereas HIF1 $\alpha$, when activated, functions as a nuclear transcription factor (18).

To test the hypothesis that MAOA regulates HIF1 $\alpha$ stability by directly affecting PHD activity, we determined PHD activity in control and MAOA-overexpressing PC-3 cells by measuring hydroxylated HIF $1 \alpha$ level, and these cells were pretreated with the proteasomal inhibitor MG132 to prevent hydroxylated HIF1 $\alpha$ from being degraded. More HIF1 $\alpha$ but significantly less hydroxylated HIF1 $\alpha$ was accumulated during MG132 treatment in MAOA-overexpressing cells (Figure 3A), suggesting lower PHD activity in the presence of MAOA. The hypothesis that MAOA regulates HIF1 $\alpha$ through the PHDs was further validated by determination of the effect of treatment with dimethyloxalylglycine (DMOG), a potent PHD inhibitor, on HIF $1 \alpha$ protein stability and the expression of HIF1 $\alpha$ target genes in MAOA-overexpressing cells. If MAOA affects HIF1 $\alpha$ stability by modulating PHD activity, DMOG treatment would overcome the effects of MAOA overexpression and produce equivalent levels of HIF1 $\alpha$ stabilization in control and MAOA-overexpressing cells. Indeed, the control and MAOA-overexpressing cells demonstrated equal levels of HIF1 $\alpha$ stabilized in response to DMOG treatment (Figure 3F, lane 3 vs. lane 7). Moreover, DMOG treatment relatively reduced the induction of the HIF $1 \alpha$ target genes VEGFA and GLUT1 in MAOA-overexpressing cells compared with the control cells (Figure 3B). These data support a model in which PHD activity is already reduced in MAOA-overexpressing 
A

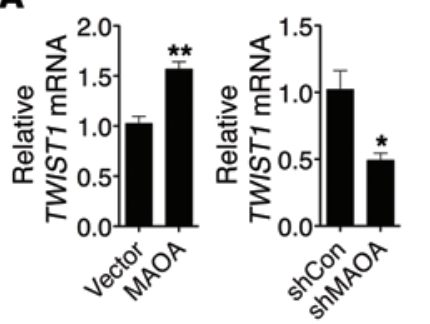

C

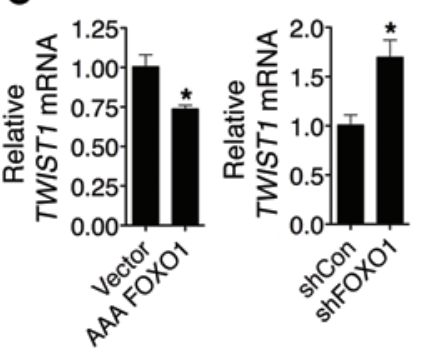

$\mathbf{F}$

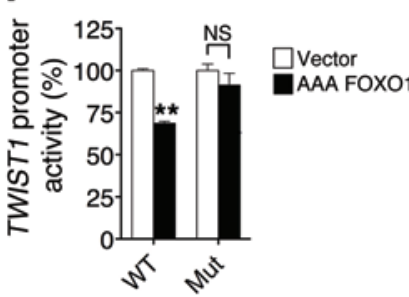

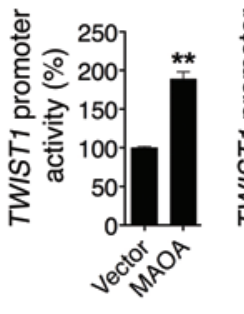

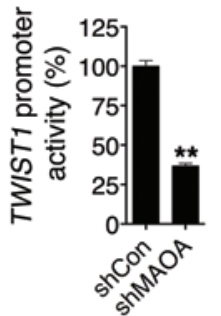

B

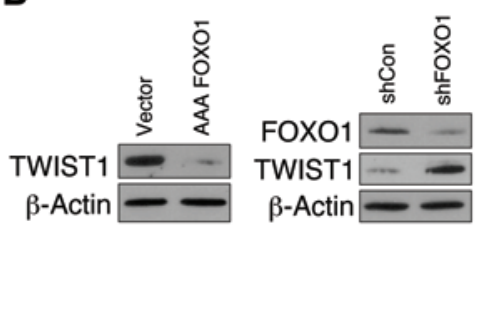

D

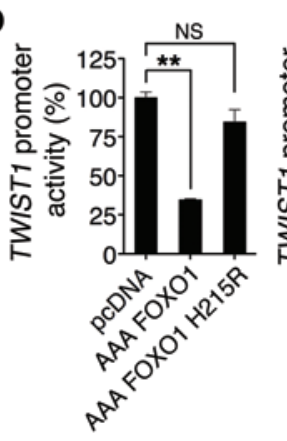

G

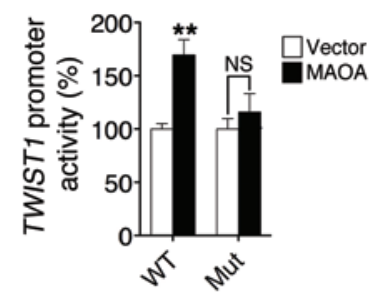

E

Canonical FOXO1-binding site

C/GA/TAAAC/AA

Potential FOXO1-binding site in TWIST1 promoter CCAAACT $(-56 /-50)$

Mutated FOX01-binding site in TWIST1 promoter

CCGCECT $(-56 /-50)$

$\begin{array}{lll}\text { Species } & & \\ \text { Homo sapiens } & -66 & \text { actgctgcccCCAAACTttccgcctgc } \\ \text { Pan troglodytes } & -117 \text { aaatatagaCCAAACTctaaggttct } \\ \text { Mus musculus } & -81 \text { accgctgccCCAAACTttccgcccgc } \\ \text { Rattus norvegicus } & -211 \text { actgctgccCCAAACTttccgccgc } \\ \text { Bos taurus } & -259 \text { actgctgccCCAAACTttccgcttgc }\end{array}$

Species

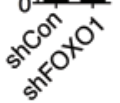

-259 actgctgccCCCAAACTttccgcttgC

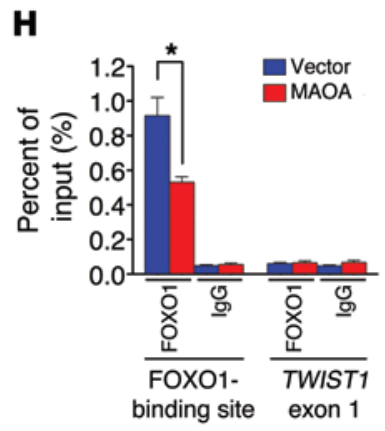

Figure 5

MAOA activates TWIST1 by reducing FOXO1 activity. (A) qPCR analysis of TWIST1 mRNA expression (mean \pm SEM, $n=3$ ) in paired PC-3 and LNCaP cells (left). A human TWIST1 promoter reporter construct was transfected into these cells, and the luciferase activity (mean \pm SEM, $n=3$ ) was assayed (right). (B) Immunoblots of paired PC-3 cells for TWIST1. AAA FOXO1 and shFOXO1 indicate a constitutively active form of $F O X O 1$ expression construct and FOXO1-targeting shRNAs, respectively. (C) qPCR analysis of TWIST1 mRNA expression (mean \pm SEM, $n=3$ ) in different pairs of PC-3 cells. (D) Determination of TWIST1 promoter activity (mean \pm SEM, $n=3$ ) in different groups of PC-3 cells as indicated. AAA FOXO1 H215R is defective in DNA-binding ability. (E) Top box: The canonical sequence of the FOXO1-binding site (top), a potential FOXO1-binding site in the TWIST1 promoter (middle), and introduced point mutations (bottom, italic and red) used to inactivate the potential FOXO1-binding site are shown. Bottom box: Alignment of the conserved FOXO1-binding site (bold) in the TWIST1 promoter across different species is shown, with the number indicating the distance from transcription initiation sites. (F and G) Determination of WT and mutated (Mut) TWIST1 promoter activity (mean \pm SEM, $n=3$ ) in different pairs of PC-3 cells. (H) ChIP analysis of PC-3 (vector and MAOA-overexpression) cells immunoprecipitated by anti-FOXO1 or IgG antibody followed by qPCR using 2 primer sets for the FOXO1-binding site in the TWIST1 promoter and TWIST1 exon 1, respectively. Data represent the percent of input (mean \pm SEM, $n=3$ ). ${ }^{*} P<0.05,{ }^{* *} P<0.01$.

cells. Consequently, when PHD activity is potently blocked by DMOG, MAOA-overexpressing cells have a smaller change in PHD activity and, thus, a smaller fold induction of HIF1 $\alpha$ target genes. To determine which isoforms of PHDs are involved in MAOA regulation of HIF1 $\alpha$, the protein expression of all 4 PHD isoforms was measured in hypoxia-treated MAOA-overexpressing PC-3 cells. Specifically, PHD3 was repressed by MAOA overexpression at both protein and mRNA levels, whereas the expression of other PHDs remained unchanged (Figure 3C and Supplemental Figure 7A). Moreover, genetic knockdown of PHD3 but not other PHDs via siRNA recapitulated DMOG effects on HIF1 $\alpha$ target gene expression in MAOA-overexpressing cells compared with control cells
(Supplemental Figure 7, B and C). Taken together, our results suggest that MAOA-overexpressing PCa cells selectively reduced PHD3 activity, thus enhancing HIF1 $\alpha$ stability and activity, culminating in increased downstream target gene expression.

In addition to intracellular oxygen concentration, PHD activity can also be regulated by several intracellular signals, including ROS, which have been shown to inhibit the PHDs and stabilize HIF1 $\alpha$ $(15,16)$. Moreover, hypoxia enhances ROS production, a seemingly required step for the hypoxic activation of HIF1 $\alpha$ (19). Because MAOA-mediated enzymatic reactions produce hydrogen peroxide, which is responsible for the generation of ROS as a by-product (3), we hypothesized that increased ROS in MAOA-overexpressing 
cells contribute to PHD inhibition and consequent HIF1 $\alpha$ stabilization in PCa cells. As expected, MAOA overexpression increased the generation of both mitochondrial hydrogen peroxide and ROS in PC-3 cells, while these were reduced in MAOA-knockdown LNCaP cells (Supplemental Figure 8, A-D). We found further that hypoxia triggered a significantly higher increase in ROS production in MAOA-overexpressing cells than control cells (Figure 3D), providing a mechanistic explanation for why MAOA-overexpressing cells have exaggerated responses to hypoxia. We next treated cells with the antioxidant N-acetylcysteine (NAC) to suppress ROS and hence block the downstream effects of MAOA mediated potentially by intracellular ROS. Indeed, the higher HIF $1 \alpha$ level in hypoxia-treated MAOA-overexpressing cells could be significantly attenuated by NAC treatment (Figure 3E, lane 8 vs. lane 7). Moreover, when PHD activity was blocked by DMOG treatment, control and MAOA-overexpressing cells both demonstrated comparable HIF1 $\alpha$ level, and NAC failed to destabilize HIF1 $\alpha$ (Figure 3F). Consistent with decreased HIF1 $\alpha$ expression in NACtreated MAOA-overexpressing cells, the expression of the HIF1 $\alpha$ target genes VEGFA, GLUT1, and TWIST1 was reduced to control levels in NAC-treated MAOA-overexpressing cells (Figure 3G). Furthermore, NAC treatment of cells reversed MAOA-induced EMT characteristics by decreasing mesenchymal marker expression and cell migratory and invasive behaviors (Supplemental Figure 5, D and E). To test whether increased ROS contribute to the proliferation of MAOA-overexpressing cells, we determined the growth profiles in cells cultured with NAC. Strikingly, we observed that NAC blunted the enhanced proliferation in MAOA-overexpressing cells under both normoxic and hypoxic conditions, restoring their growth rates to that of the control cells (Figure $3 \mathrm{H}$ ). Together, MAOA regulation of ROS augmented hypoxic responses by increasing the steady-state HIF $1 \alpha$ level and its target gene expression, including VEGFA, GLUT1, and TWIST1, which converge the cell signaling network toward a mesenchymal transition and enhanced cell proliferation in MAOA-overexpressing cells (Figure 3I).

$M A O A$ activates $V E G F-A /$ neuropilin-1 signaling and its downstream AKT/FOXO1 pathway. Given that hypoxia reportedly can affect cell behavior by promoting EMT, we hypothesized that the HIF1 $\alpha$ target gene VEGFA in conjugation with its receptor(s) may play a crucial mediating role in controlling the downstream signaling for the MAOA-induced EMT and associated increased aggressive phenotype in PCa cells. VEGF has been implicated in poor prognosis and survival in PCa patients, and an elevated level of VEGF could be the trigger for an angiogenic switch in the lethal progression of advanced $\mathrm{PCa}$ (20). VEGFA expression was assessed in control/MAOA-overexpressing PC-3 or control/MAOA-knockdown LNCaP cells by quantitative PCR (qPCR), and there was a positive correlation between VEGFA and MAOA expression (Figure $4 \mathrm{~A}$, left). We further quantified VEGF-A expression in the culture media of MAOA-manipulated PCa cells by ELISA and confirmed that MAOA upregulated expression of VEGF-A and its extracellular secretion (Figure 4A, right). By contrast, we did not observe any VEGF effect on MAOA gene expression (Supplemental Figure 9, A-C). Autocrine VEGF signaling in tumor cells can occur through a nonangiogenic cell proliferative mechanism via increased autocrine VEGF-A and interaction with its coreceptor neuropilin-1 (NRP1), and such interaction has been implicated in cancer cell autonomy and aggressive behaviors (21). We showed that overexpression of MAOA significantly increased NRP1 expression in PC-3 cells (Figure 4B), which was further demonstrated in parallel in MAOA-overexpressing PC-3 xenograft tumors with concurrently increased VEGF-A expression (Figure 4C).

To elucidate the mechanism(s) by which MAOA activation of VEGF-A and NRP1 mediates downstream signaling to promote EMT, select kinases that are implicated in PCa progression were examined in PCa cells previously subjected to MAOA manipulation. As shown in Figure 4D (left panel), there was a significant increase in the relative phosphorylation of both AKT and its downstream target FOXO1 by MAOA overexpression. AKT plays a central role by activating its many downstream target genes in regulating $\mathrm{PCa}$ initiation and progression (22). FOXO1, a member of the $\mathrm{O}$ subclass of the forkhead family of transcription factors, can be regulated via the phosphorylation of AKT upon PI3K/AKT signaling activation, and has demonstrated proapoptotic function in a variety of cancers (23). We further showed that AKT/FOXO1 signaling was conversely attenuated in MAOA-knockdown LNCaP cells (Figure 4D, right panel). These results suggest that the AKT/ FOXO1 signaling pathway is downstream of MAOA.

Given that NRP1 can regulate AKT activity (24), which was reproduced in NRP1-overexpressing PC-3 cells with concurrent induction of EMT and cell proliferation (Supplemental Figure 10, A-D), we examined autocrine VEGF/NRP1-mediated signaling and evaluated the relationship between MAOA and AKT/FOXO1 signaling in PC-3 cells with stable knockdown of NRP1 using a lentiviral shRNA-mediated approach. As shown in Figure 4E, NRP1 knockdown significantly reduced both the basal and MAOA-induced phosphorylation of AKT and FOXO1 in PC-3 cells. Moreover, we demonstrated that MAOA facilitated the nuclear export of FOXO1 by altering the ratios of FOXO1 toward enhanced levels in the cytoplasmic fraction (Figure 4F, left panel), and this was confirmed by the IHC staining pattern of FOXO1 and phospho-FOXO1 in control and MAOA-overexpressing PC-3 tumor xenograft samples (Supplemental Figure 4E). Conversely, genetic silencing of NRP1 increased nuclear but decreased cytoplasmic FOXO1 expression (Figure 4F, right panel). To determine whether VEGF-A acts as the major ligand to mediate NRP1 effect in the MAOA context, we treated control and MAOA-overexpressing PC-3 cells with antiVEGF-A antibody. This targeted blockade of receptor binding of secreted VEGF-A attenuated the MAOA-induced phosphorylation of both AKT and FOXO1 toward that of the control cells (Figure 4G). Unlike VEGF-A, we found that another potential NRP1 receptor ligand, semaphorin $3 \mathrm{~A}$ (SEMA3A), was not elevated in MAOA-overexpressing PC-3 cells (Supplemental Figure 11), further confirming the mediating role of VEGF-A in controlling the MAOA/NRP1 axis. By examining the functional roles of NRP1 in determining cell behavior, we showed that NRP1 knockdown resulted in a significant decrease of MAOA-induced migration and invasion in PC-3 cells (Figure 4H). Likewise, NRP1 knockdown also dramatically reduced the cell proliferation rate in MAOA-overexpressing cells to a level lower than that of the control (Figure 4I). Taken together, these functional characteristics indicate that the VEGF-A/NRP1 system mediates MAOA-induced EMT and cell proliferation via the downstream AKT/FOXO1 signaling.

TWIST1 expression is directly regulated by transcription factor FOXO1. We screened a spectrum of key transcription factors known to promote EMT in our present model system, and identified TWIST1 as 1 of the downstream targets of MAOA (Figure 1B). TWIST1, a basic helix-loop-helix transcription factor, is a master regulator of EMT, and its expression in tumors often correlates with aggressive disease and poor outcome $(25,26)$. We showed that genetic silencing of 

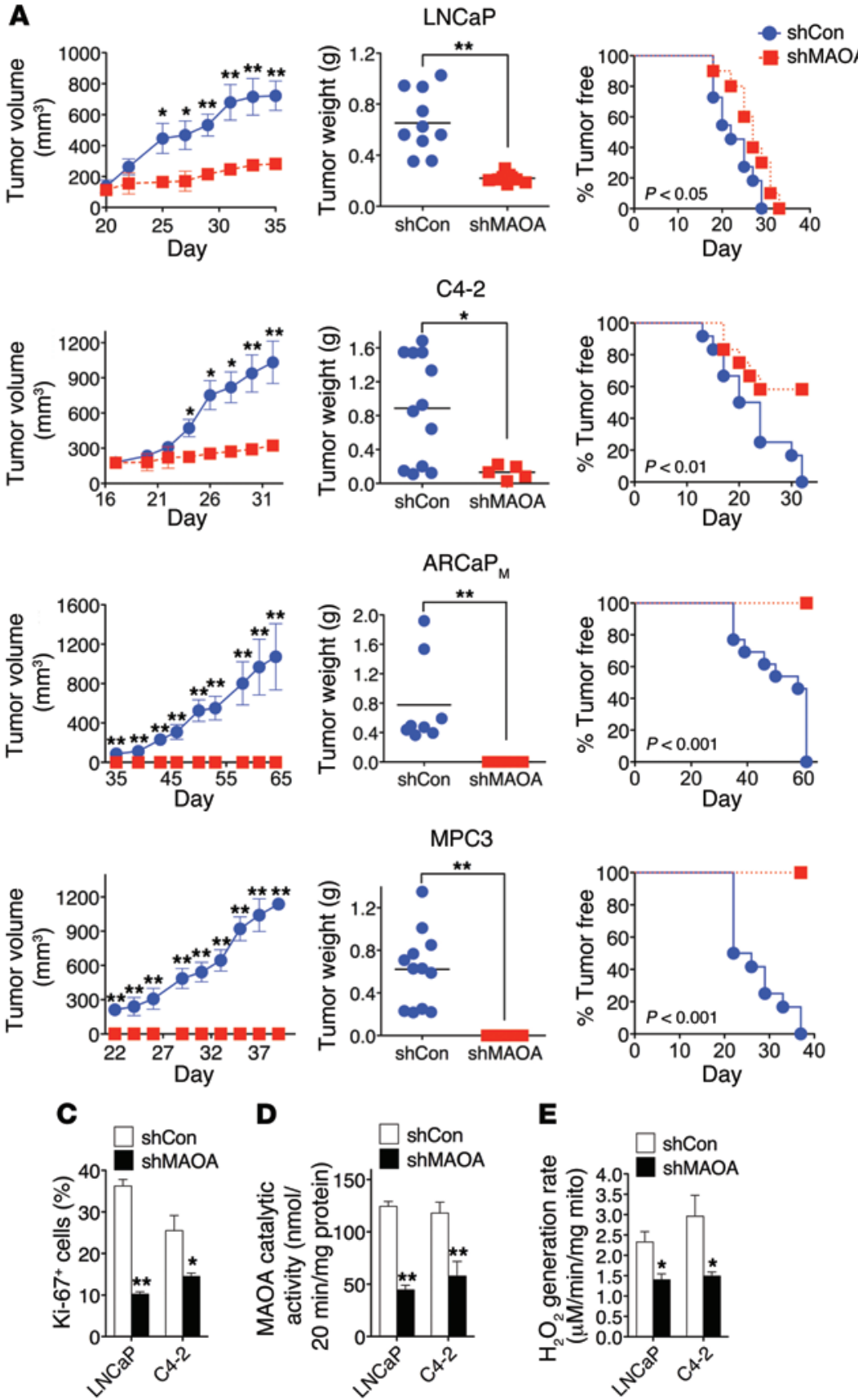

E

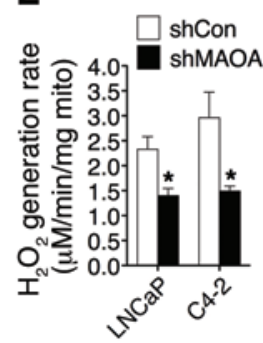

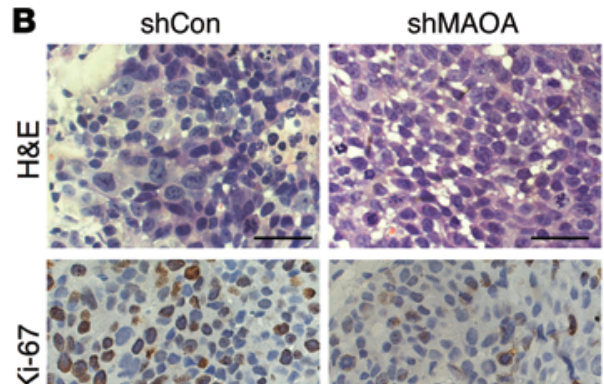
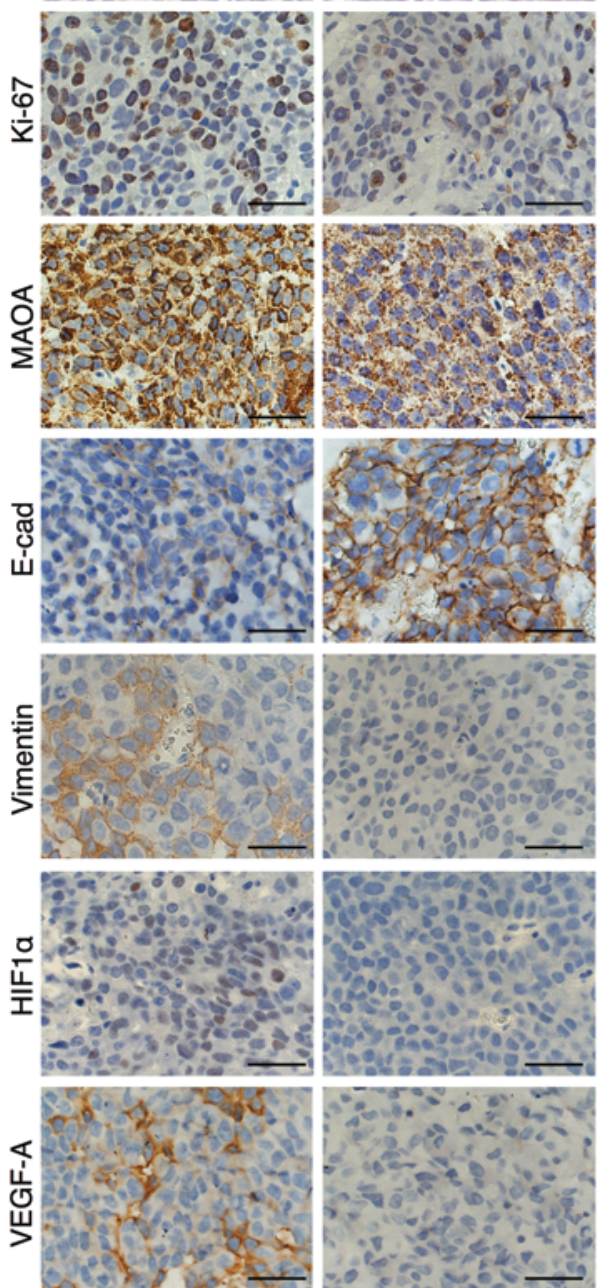

Figure 6

MAOA is essential for the growth of prostate tumor xenografts by regulating EMT, hypoxia, and ROS. (A) LNCaP, C4-2, ARCaP $\mathrm{M}$, or MPC3 cells that stably express a MAOA-targeting shRNA (shMAOA) or a scrambled shRNA (shCon) were injected s.c. into male nude mice ( $n=4-7$ mice for each group; details are given in Supplemental Table 1) for the growth of tumor xenografts. Tumor growth was determined by measurement of tumor volume, tumor weight, and the frequency of tumor formation. The graphs show the mean $( \pm \mathrm{SEM})$ tumor size and tumor-free percentages at the indicated times. ${ }^{*} P<0.05$, ${ }^{*} P<0.01$. (B) H\&E and IHC analysis of Ki-67, MAOA, E-cadherin, vimentin, HIF1 $\alpha$, and VEGF-A expressions in LNCaP (shCon and shMAOA) tumor xenografts. Representative images from 5 separate samples are shown. Original magnification, $\times 400$; scale bars: $20 \mathrm{\mu m}$. (C) Quantification of percentage of Ki-67+ tumor cells in paired LNCaP and C4-2 tumor xenografts from 5 distinct images of each tumor sample ( $n=5$ tumor samples for each group). Data represent the mean \pm SEM. ${ }^{*} P<0.05$, ${ }^{* *} P<0.01$. (D) Determination of MAOA enzymatic activity in paired LNCaP and C4-2 tumor xenografts. Data represent the mean \pm SEM from all tumors obtained at mouse necropsy ( $n=5-12$ tumors for each group; details are given in Supplemental Table 1). ${ }^{* *} P<0.01$. (E) Determination of $\mathrm{H}_{2} \mathrm{O}_{2}$ generation rate in intact mitochondria isolated from paired LNCaP and C4-2 tumor xenografts ( $n=3$ tumor samples for each group) by Amplex Red hydrogen peroxide assay. Data represent the mean \pm SEM. ${ }^{*} P<0.05$. 
A

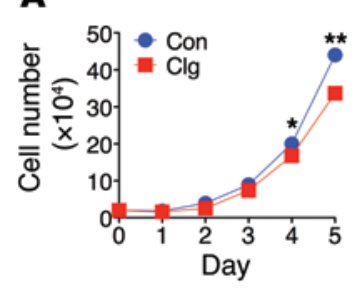

E

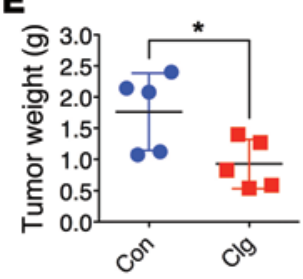

B

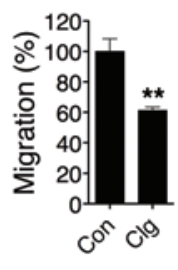

$\mathbf{F}$

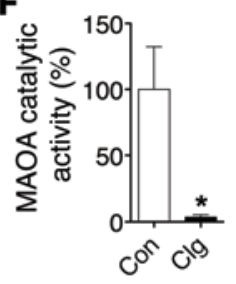

C

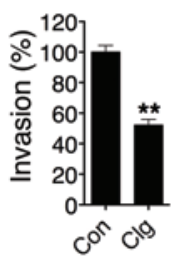

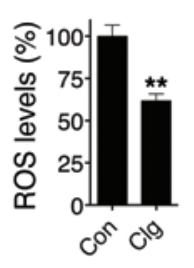

D

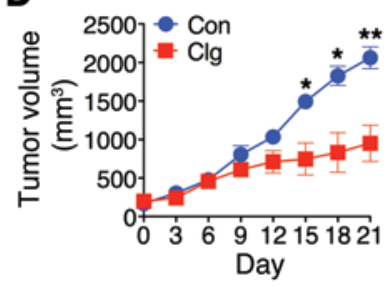

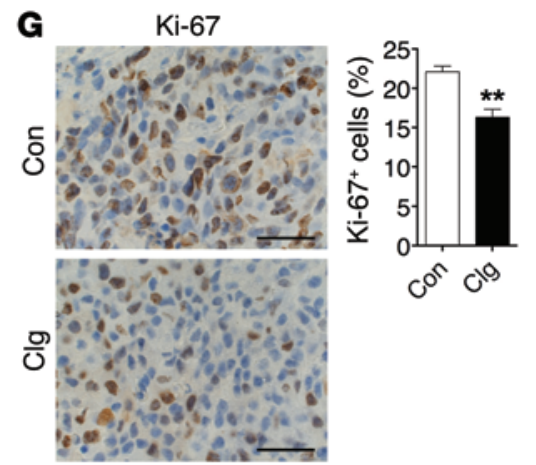

H

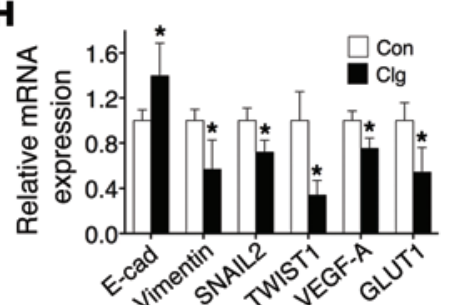

\section{Figure 7}

Clorgyline inhibits the growth of PCa cells and tumor xenografts. (A) Growth curves of LNCaP cells treated with either clorgyline $(1 \mu \mathrm{M}$, Clg) or vehicle (PBS, Con) for 5 days (mean \pm SEM, $n=3$ ). ${ }^{*} P<0.05,{ }^{* *} P<0.01$. (B) LNCaP cells treated with either clorgyline $(1 \mu \mathrm{M})$ or vehicle were assessed for their ability to either migrate (left) or invade (right) (mean $\pm \mathrm{SEM}, n=3$ ). ${ }^{\star *} P<0.01$. (C) Determination of ROS levels in LNCaP cells treated with clorgyline ( $1 \mu \mathrm{M}, 48$ hours) or vehicle (mean $\pm \mathrm{SEM}, n=3$ ). ${ }^{*} P<0.01$. (D) Determination of the growth of C4-2 subcutaneous tumor xenografts in mice ( $n=5$ mice for each group) treated with clorgyline (10 mg/kg, i.p.) or saline on a daily basis for 21 days. The graph shows the mean ( \pm SEM) tumor size at the indicated time. ${ }^{*} P<0.05,{ }^{*} P<0.01$. (E) Determination of tumor weight at mouse necropsy. ${ }^{*} P<0.05$. (F) Determination of MAOA enzymatic activity (mean \pm SEM) in clorgyline- or vehicle-treated tumor xenografts $(n=5$ tumor samples for each group). ${ }^{*} P<0.05$. (G) Quantification of percent of Ki-67+ (mean $\pm \mathrm{SEM}$ ) tumor cells in clorgyline- or vehicle-treated tumor xenografts from 5 distinct images of each tumor sample ( $n=5$ tumor samples for each group) (right). Representative IHC images are shown (left). ${ }^{* *} P<0.01$. Original magnification, $\times 400$; scale bars: $20 \mu \mathrm{m}$. (H) qPCR analysis of clorgyline- or vehicle-treated tumor xenografts $(n=5$ tumor samples for each group) for the expression of EMT marker and HIF1 $\alpha$ target genes. Data represent the mean \pm SEM. ${ }^{*} P<0.05$.

TWIST1 reversed MAOA-induced EMT by decreasing mesenchymal marker expression and cell migratory, invasive, and proliferative behavior (Supplemental Figure 12, A-C), which suggests that TWIST 1 is functional for the induction of EMT by MAOA. To assess the potential regulation of TWIST1 by MAOA, we determined both TWIST1 mRNA and its promoter activity in PCa cells with manipulated MAOA expression. Overexpression of MAOA increased TWIST1 mRNA and promoter activity in PC-3 cells, whereas these TWIST1-related activities were reduced upon MAOA knockdown in LNCaP cells (Figure 5A), suggesting that MAOA may regulate TWIST1 expression at the transcriptional level.

AKT drives EMT and cancer progression by regulating many downstream target genes, including TWIST1 $(27,28)$, but the specific AKT-responsive signaling pathways that regulate TWIST1 remain to be clarified. The transcription factor forkhead box (FOX) proteins capable of being phosphorylated by AKT play a dominant role in cancer progression (23). Moreover, FOX proteins have been demonstrated to mediate EMT by regulating EMTinducing factors (29), which led us to speculate that FOXO1 may regulate TWIST1 in PCa cells. We established PC-3 cells that stably overexpressed a constitutively active FOXO1 expression construct (AAA FOXO1) with 3 mutated phosphorylation sites (T24A, S256A, and S319A). AAA FOXO1 is sequestered in the nucleus without degradation (30). Enforced expression of AAA FOXO1 significantly reduced TWIST1 protein expression, whereas sta- ble shRNA-mediated silencing of FOXO1 increased TWIST1 levels in PC-3 cells (Figure 5B), suggesting a negative regulatory link between these 2 proteins. We also assessed whether FOXO1 directly participates in EMT induced by MAOA. We showed that stable knockdown of FOXO1 in MAOA-overexpressing cells further enhanced EMT characteristics by increasing mesenchymal marker expression and corresponding cell behavior including migration, invasion, and proliferation (Supplemental Figure 12, D-F), which provides a functional basis underlying the FOXO1 repression of TWIST1.

Considering the innate feature of FOXO1 as a transcription factor, we next determined whether FOXO1 transcriptionally regulates TWIST1 expression, and we showed a reproducible negative regulation of TWIST1 mRNA by FOXO1 in PC-3 cells (Figure 5C). To explore the possible direct influence of FOXO1 on the promoter of the TWIST1 gene, a 1-kb DNA segment located upstream of the transcription initiation site of TWIST1 was introduced as a luciferase reporter construct into PC-3 cells (31). Since FOXO1 can affect the transcription of target genes either through direct binding to their consensus DNA sequences in the promoter or via indirect protein-protein interactions with other transcription factors or cofactors (32), we distinguished these 2 alternative mechanisms by using a FOXO1 mutant (AAA FOXO1 H215R) that is deficient in DNA binding (30). In contrast to its WT counterpart, which strongly repressed TWIST1 promoter activity, the FOXO1 mutant 

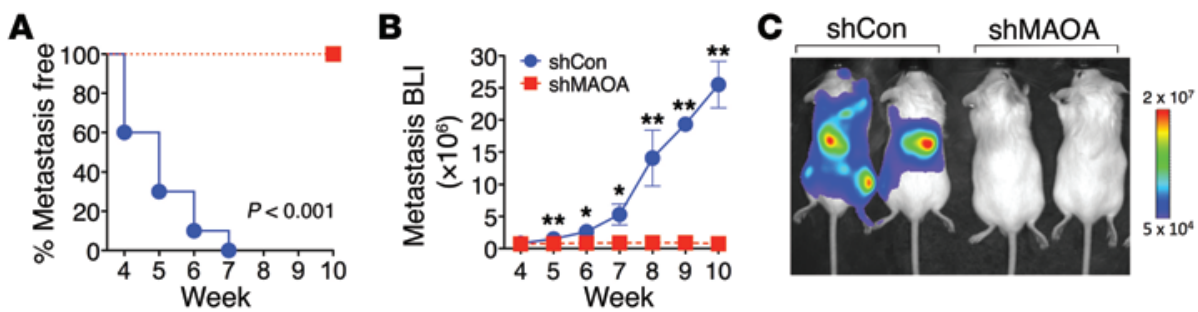

Figure 8

MAOA is essential for PCa metastasis. (A) Kaplan-Meier curves of metastasis development of control (shCon) and MAOA-knockdown (shMAOA) $\mathrm{ARCaP}_{\mathrm{M}}$ cells ( $n=10$ mice for each group). (B) Normalized BLI curves of metastasis development for each experimental group. Data represent the mean \pm SEM $(n=10) .{ }^{\star} P<0.05,{ }^{* \star} P<0.01$. (C) Representative BLI (week 10) images of 2 of 10 mice in each group from $\mathbf{B}$.

failed to do so (Figure 5D, left panel). Hence, the FOXO1 action on the TWIST1 promoter appeared to require the intact DNA-binding activity of FOXO1. This regulation is further supported by the observation that genetic silencing of FOXO1 increased TWIST1 promoter activity in PC-3 cells (Figure 5D, right panel).

It remained unclear whether the observed repression is mediated by direct binding of FOXO1 to the TWIST1 promoter. To address this issue, we attempted to identify a FOXO1-response element in the TWIST1 promoter. Serial deletion of the TWIST1 promoter combined with sequence analysis revealed a region within the TWIST1 promoter $(-56 /-50)$ that exhibits strong sequence similarity to the canonical AT-rich FOXO1-binding site (Figure 5E, top panel) $(33,34)$. To test whether the potential direct regulation of TWIST1 by FOXO1 is conserved throughout evolution, we searched for the consensus FOXO1-binding site and its surrounding sequences in the human TWIST1 promoter across species. Interestingly, we found that this element (CCAAACT) is highly conserved among amniotic genomes examined, including chimpanzees, mice, rats, and cows (Figure 5E, bottom panel). Accordingly, we generated a mutant TWIST1 promoter reporter construct harboring 3 point mutations in the center of the putative FOXO1response element. The resulting mutated TWIST1 promoter was no longer repressed by ectopic expression of FOXO1 in PC-3 cells (Figure 5F). This TWIST1 promoter reporter mutant construct was further introduced into MAOA-overexpressing PC-3 cells, and in contrast to its WT counterpart, the mutant did not respond to MAOA overexpression (Figure 5G). To confirm the direct occupancy of FOXO1 with the sequences in the TWIST1 promoter in vivo, we extended these studies by performing ChIP analysis. We isolated chromatin-nuclear protein complexes immunoprecipitated with anti-FOXO1 antibody from both control and MAOA-overexpressing PC-3 cells, and analyzed it by qPCR using primers that specifically encompass the putative FOXO1-response element in the TWIST1 promoter. We were able to detect the physical association of FOXO1 with the TWIST1 promoter sequences, and such association was reduced in the presence of MAOA overexpression (Figure $5 \mathrm{H}$ ), which is consistent with the observation of less nuclear FOXO1 expression in MAOA-overexpressing PC-3 cells (Figure 4F, left panel). Moreover, limited signals were detected from the negative controls, in which either nonspecific IgG antibody was used in the immunoprecipitation step or the TWIST1 exon 1 was probed in order to confirm the targeting specificity of the primer set used in PCR. These results in sum provide evidence that MAOA negatively mediates TWIST1 gene activation via direct interaction of FOXO1 with a specific element located in the TWIST1 promoter. In the case of MAOA overexpression in PCa cells, increased TWIST1 gene activation that drives EMT was the result of sequestration of FOXO1 from nucleus to cytoplasm, reducing the overall suppression of TWIST1 gene expression.

$M A O A$ function in prostate tumorigenesis and cancer metastasis. The MAOA effects on promotion of EMT, hypoxia, and ROS production described above shed light on how MAOA supports PCa growth and development. To test this hypothesis, we established multiple prostate tumor xenograft mouse models to determine the functional roles of MAOA in controlling tumor growth (Supplemental Table 1). We used 3 human PCa cell lines that show different MAOA expression levels, aggressiveness, and invasiveness as well as other innate features such as responses to androgen $(35,36)$. Considering the importance of the immune system in PCa growth and progression, we also extended our analysis by using a highly tumorigenic mouse prostate carcinoma MPC3 cell line in an immunocompetent mouse model. The MPC3 line was derived from mouse primary prostate tumors harboring double loss of Pten and p53 tumor suppressors. To assess MAOA function in these tumor xenograft models, we first infected these cells with a lentiviral construct expressing an shRNA directed against the mRNA encoding MAOA, or a control that targets no known mammalian genes. Stable introduction of MAOA-specific shRNAs decreased MAOA enzymatic activity by more than $50 \%-70 \%$ in all cell lines (Supplemental Figure 13A). In line with the observation that enforced MAOA expression significantly induced PC-3 cell proliferation (Supplemental Figure 4A), cells expressing MAOA-targeting shRNAs had consistently reduced cell proliferation rates in comparison with control cells (Supplemental Figure 13B). In these studies, we observed no significant apoptotic changes in either MAOA-overexpressing PC-3 or MAOA-knockdown LNCaP cells compared with their respective control cells (Supplemental Figure 14, A and B). These complementary results suggest that MAOA affects PCa cell growth mainly through its proliferation-enhancing functions.

After being implanted s.c. into male nude mice, LNCaP and its lineage-derived androgen-independent C4-2 cells expressing $M A O A$-targeting shRNAs showed slower tumor growth rates and formed fewer tumors than controls. Cells stably expressing $M A O A$-targeting shRNA that did form tumors formed tumors that were quite small, with an average tumor weight of $221 \pm 36 \mathrm{mg}$ and $132 \pm 81 \mathrm{mg}$ for LNCaP and C4-2 tumors, respectively, compared with larger tumors, with an average weight of $653 \pm 232 \mathrm{mg}$ and $888 \pm 632 \mathrm{mg}$ for LNCaP and C4-2 control tumors, respectively (Figure 6A). Strikingly, stable knockdown of MAOA in $\mathrm{ARCaP}_{\mathrm{M}}$, 
A
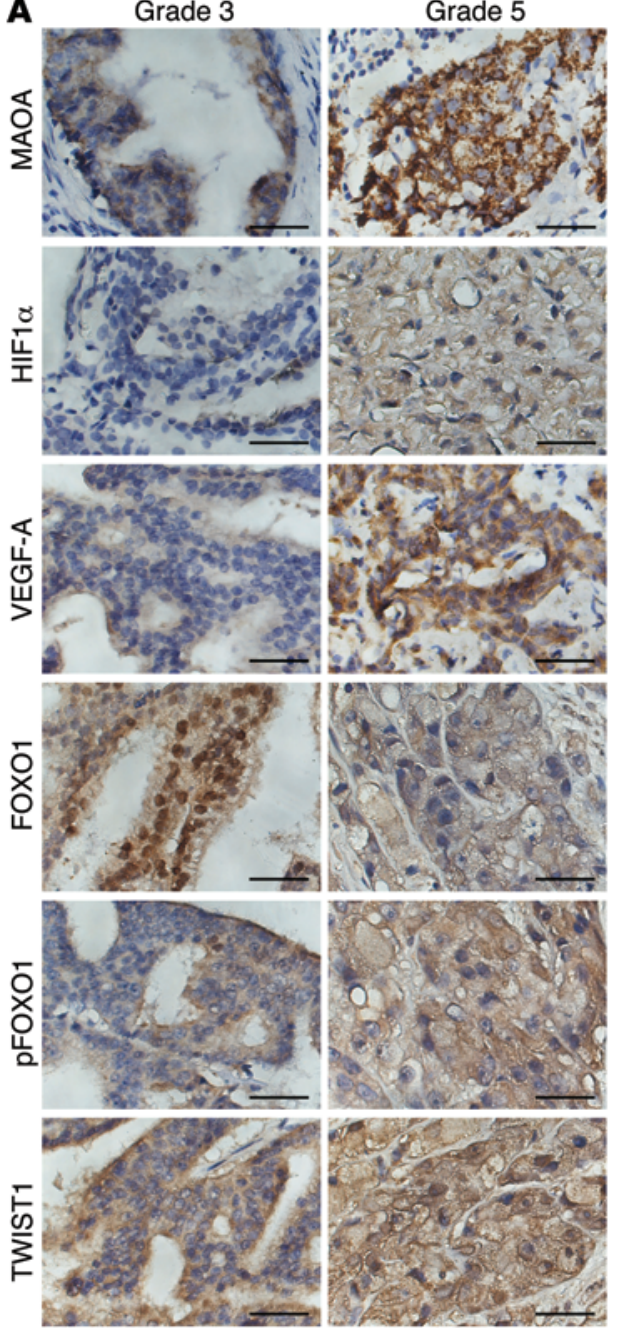

B
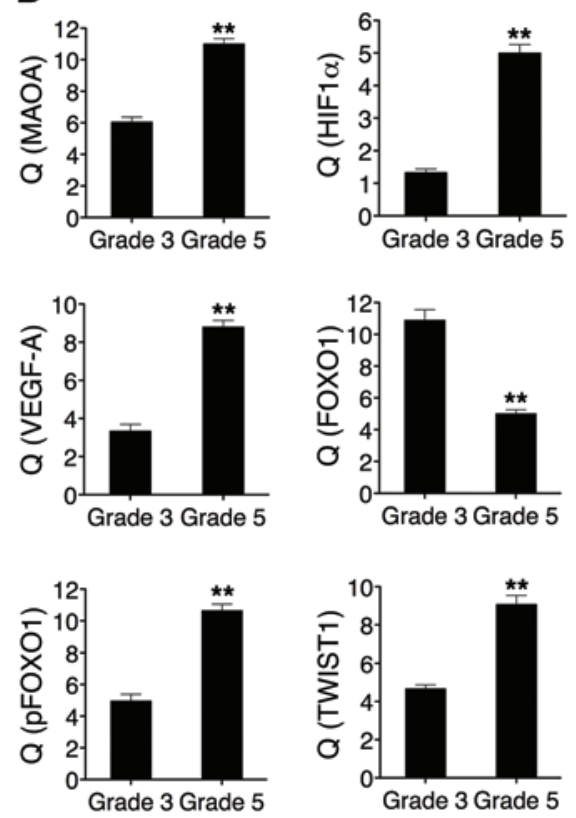

Figure 9

The HIF1 $\alpha /$ VEGF-A/FOXO1/TWIST1 pathway is manifested in high-Gleason grade $\mathrm{PCa}$. (A) Sixty specimens of human $\mathrm{PCa}$ including 30 Gleason grade 3 tumors and 30 Gleason grade 5 tumors were immunostained for MAOA, HIF1 $\alpha$, VEGF-A, FOXO1, pFOXO1, and TWIST1. Original magnification, $\times 400$; scale bars: $20 \mu \mathrm{m}$. (B) Semiquantitative analysis of $\mathrm{IHC}$ staining was performed for all specimens to assess both the percentage of cells stained and the intensity of each staining. This analysis is reported as the quotient $(Q)$ of these 2 parameters (mean \pm SEM). Details are given in Supplemental Methods. Images representative of the mean $Q$ for each IHC staining are shown. ${ }^{\star \star} P<0.01$. an aggressive human PCa cell line, and MPC3 cells completely eliminated their in vivo growth, in sharp contrast to the expected explosive tumor growth in mice inoculated with the control cells.

As a comparison, we next analyzed the protein expression patterns of select markers in tumor specimens from LNCaP and C4-2 xenograft tumors by IHC. Ki-67 staining of tumor specimens revealed a $40 \%-70 \%$ decrease of $\mathrm{Ki}-67^{+}$cells in the MAOA-knockdown group of both LNCaP and C4-2 tumors (Figure 6, B and C). MAOA protein staining also showed decreased intensity in MAOAknockdown tumor samples for both lines (Figure 6B), which is consistent with the results of reduced MAOA enzymatic activity in harvested tumors expressing MAOA-targeting shRNAs (Figure $6 \mathrm{D}$ ), supporting the concept that genetically silencing $M A O A$ gene expression by an shRNA-mediated protocol is highly effective and sustainable under in vivo conditions. Moreover, MAOA-knockdown xenograft tumors showed EMT reversal by increased staining of E-cadherin as well as reduced expression of vimentin, and repressed tumor hypoxia by less HIF1 $\alpha$ and VEGF-A staining (Figure $6 \mathrm{~B}$ and Supplemental Figure 15). These results from tumor xenograft mouse models provide further evidence that MAOA drives EMT and augments PCa cell response to hypoxia.

We further examined the levels of ROS in both control and MAOA-knockdown tumor samples. MAOA is located in the outer membrane of mitochondria and directly engaged in hydrogen peroxide production via the oxidative deamination of its substrates, which can be subsequently converted into other forms of ROS (3). We determined the rate of hydrogen peroxide generation in intact tumor mitochondria, which represents specifically the differences in MAOA-originated ROS production, from both control and MAOA-knockdown tumors. LNCaP and C4-2 tumors that expressed MAOA-targeting shRNAs showed slower rates of hydrogen peroxide generation in comparison with control tumors (Figure 6E), suggesting that increased ROS production could be a crucial factor underlying MAOA's role in PCa development, in line with in vitro studies.

We next evaluated the therapeutic effect of small-molecule inhibitors of MAOA capable of interfering with MAOA enzymatic activity in PCa xenograft mouse models. Treatment of LNCaP cells with clorgyline, a potent MAOA inhibitor (37), decreased cell proliferation, migration, and invasion (Figure 7, A and B), and suppressed cellular ROS levels (Figure 7C). Mice bearing subcutaneously androgen-independent and aggressive C4-2 xenograft tumors (36, 38), when receiving clorgyline treatment via the i.p. route, showed reduced tumor growth by both slower growth rate and smaller tumor weight within a 3-week treatment period (Figure 7, D and E). In the meanwhile, we did not observe significant differences of 


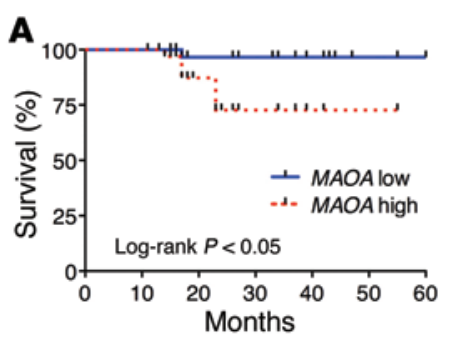

B
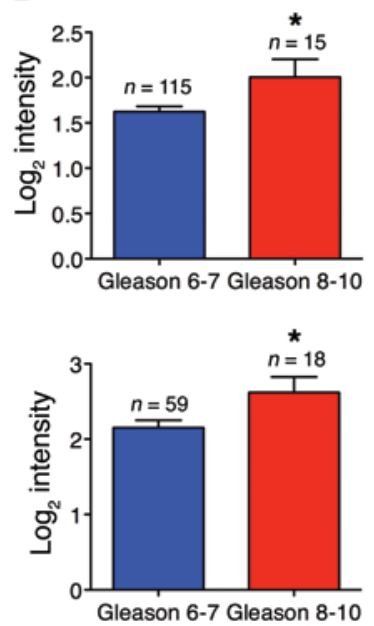

Taylor 3
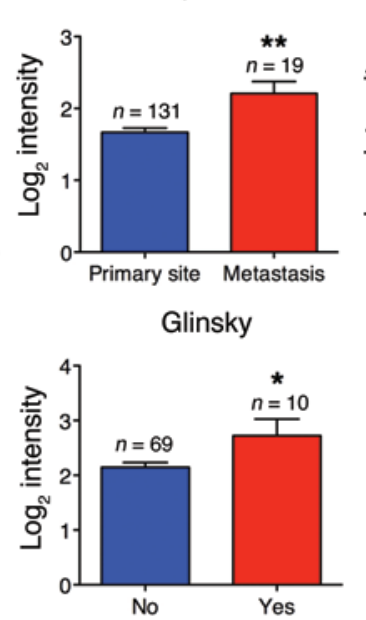
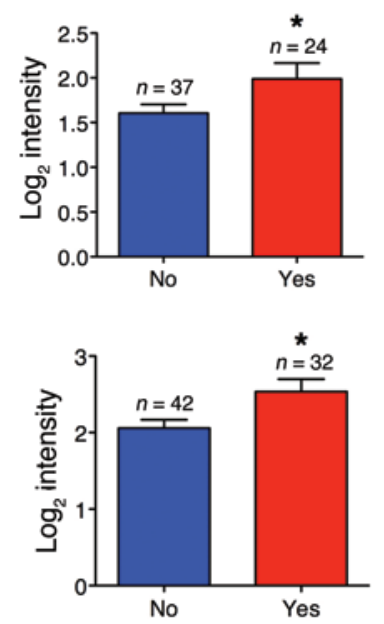

\section{Figure 10}

Increased MAOA expression is associated with poor prognosis in PCa patients. (A) Kaplan-Meier plots of survival of PCa patients stratified by the expression of MAOA from tissue microarrays (74 patients in total; MAOA-low, $n=37$; MAOA-high, $n=37$ ). Detailed patient information is given in Supplemental Table 3. The $P$ value was calculated by a log-rank test. (B) Oncomine analysis of MAOA transcript level in 2 publicly available DNA microarray data sets (top, Taylor 3; bottom, Glinsky) regarding Gleason score (Taylor 3, left; Glinsky, left), cancer sample site (Taylor 3, middle), seminal vesicle involvement (Glinsky, middle), and 5-year recurrence status (Taylor 3, right; Glinsky, right). ${ }^{\star} P<0.05,{ }^{* \star} P<0.01$. mouse body weight between the control and treatment groups. The efficacy of inhibition of MAOA enzymatic activity was confirmed in tumor samples at mouse necropsy (Figure 7F). By closely characterizing tumor samples, we demonstrated less Ki-67 staining, with a $26 \%$ drop of $\mathrm{Ki}-67^{+}$cells, in the tumor specimens of the treatment group (Figure 7G). Moreover, qPCR analysis of tumor samples further revealed consistent changes in select markers of MAOA-knockdown effects, demonstrating reduced EMT and hypoxic responses to clorgyline treatment (Figure $7 \mathrm{H}$ ).

In addition to the participation of MAOA in determining prostate tumorigenesis and cancer growth, our accumulated evidence that MAOA induced EMT and invasiveness in multiple lines of PCa cells further led us to speculate that MAOA may mediate PCa metastasis. To directly test whether MAOA is functionally important for PCa metastasis, we used $\mathrm{ARCaP}_{\mathrm{M}}$ cells, a highly metastatic human PCa cell line $(35,39)$, which expressed either a control shRNA or a $M A O A$-specific shRNA to stably silence MAOA expression. The progression of cancer metastasis after intracardiac injection of tumor cells in mice was monitored by weekly bioluminescence imaging (BLI) using a stably expressed Firefly luciferase reporter. BLI analysis showed that MAOA knockdown significantly abrogated cancer metastasis in mice within a 10-week period after injection (Figure 8, A-C). In contrast, we observed significant metastases of control cells to various organs, including bone, adrenal gland, lymph node, lung, and liver, by BLI imaging with results confirmed by routine necrotic protocols (Supplemental Table 2). Taken together, these data strongly demonstrate that MAOA is a key determinant of tumor growth and metastasis in mice, potentially coordinating the regulation of EMT, hypoxia, and oxidative stress.

The HIF1 $\alpha / V E G F-A / F O X O 1 / T W I S T 1$ pathway is manifested in highGleason grade $P C a$. A critical question that arises from our in vivo data is whether the expression of HIF1 $\alpha$, VEGF-A, and pFOXO1/ FOXO1 and the activation of TWIST1 correlate with clinical grading in human PCa, and whether the expression of these proteins correlates with MAOA in the same specimens as predicted by our hypothesis. To address this question, we used a semiquantitative analysis of IHC staining to assess the expression of these proteins in specimens from $60 \mathrm{PCa}$ patients, of which 30 were Gleason grade 3 and 30 were grade 5. Expression of MAOA was significantly higher in the cytoplasm of grade 5 compared with grade 3 PCa (Figure 9A), confirming previous studies. We observed intense widespread nuclear HIF1 $\alpha$ expression in grade 5 cells that was absent in grade 3 cells. Intense VEGF-A immunostaining was also evident in Gleason grade 5 tumor cells compared with grade 3 tumor cells (Figure 9A).

FOXO1 expression was observed in both the nucleus and cytoplasm, and there was a significant decrease of nuclear expression of FOXO1 in grade 5 tumor cells compared with grade 3 cells. pFOXO1 expression was present in both the nucleus and cytoplasm as well, but predominantly in cytoplasm. Cytoplasmic pFOXO1 showed higher staining in grade 5 cells in contrast to grade 3 cells. The differences seen in FOXO1/pFOXO1 expression patterns between Gleason grade 3 and grade 5 tumors indicate a dynamic nuclear exclusion of FOXO1 corresponding with disease progression. Moreover, intense widespread nuclear TWIST1 staining was evident in the majority of grade 5 tumor cells concurrently accompanied by a decline of nuclear FOXO1 expression (Figure 9A). These results are consistent with the hypothesis that MAOA increases TWIST1 expression by facilitating the phosphorylation and nuclear export of FOXO1 to activate the TWIST1 transcription. These IHC differences were all further confirmed by semiquantitative analysis of multiple specimens (Figure 9B). 


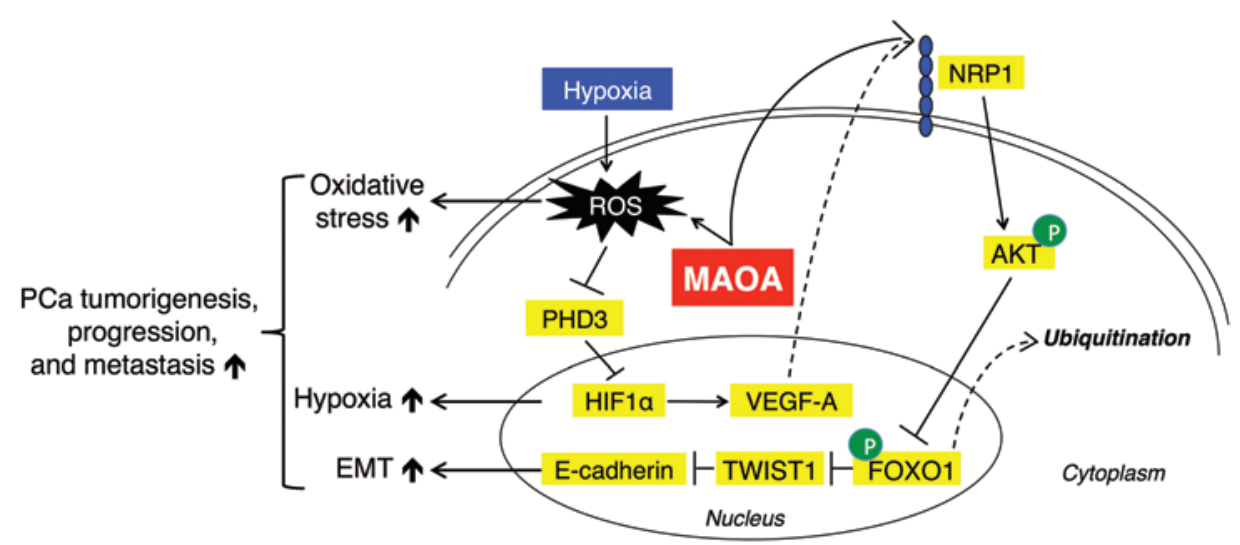

Figure 11

A proposed working model for how MAOA regulates prostate tumorigenesis, progression, and metastasis by engaging EMT, hypoxia, and ROS. MAOA induces EMT by generating ROS that inhibit PHD activity and stabilizing HIF1 $\alpha$. Stimuli, such as hypoxia, can exacerbate MAOA-mediated production of ROS. MAOA further mediates the activation of VEGF-A/NRP1 signaling to upregulate the AKT/FOXO1 pathway, which results in the nuclear export of transcription repressor FOXO1 to activate nuclear TWIST1 expression. Together, increased MAOA expression promotes EMT, hypoxia, and ROS production, which in concert drive PCa tumorigenesis, progression, and metastasis.

Increased MAOA expression is associated with poor prognosis in $\mathrm{PCa}$ patients. To determine whether high MAOA expression exhibited by high-grade $\mathrm{PCa}$ is associated with poor patient outcome, we used a tissue microarray containing 74 cases with multiple disease progression (e.g., Gleason score 6-10, T2, or T3). After semiquantitative IHC analysis of these clinical samples, the Kaplan-Meier survival curves indicated that MAOA-low patients had significantly enhanced survival times when compared with MAOA-high patients ( $\log$-rank $P=0.0267$ ) (Figure 10A and Supplemental Table 3). We further evaluated the prognostic value of $M A O A$ in multiple public clinical DNA microarray data sets using Oncomine 4.4. Considering that PCa is a biologically and clinically heterogeneous disease, we investigated whether high MAOA expression was related to multiple clinical indices in a subset of cases using Cancer Outlier Profile Analysis (COPA), a methodology that has been validated for uncovering candidate oncogenes, such as $E R G(40)$. COPA identified MAOA as significantly overexpressed in a subset of tumor samples in 15 of 27 available data sets (gene rank, top $20 \%$; fold change, $\left.>2 ; P<1 \times 10^{-4}\right)$. Using the same statistical filters, $M A O A$ displayed a COPA score comparable to or higher than that of ERG in several data sets (Supplemental Table 4).

The Gleason grading system for PCa is a key parameter for clinically assessing prognosis and choice of therapy, and cancers with a higher Gleason score are more aggressive along with a worse prognosis (13). Analysis of 2 data sets (Taylor 3 and Glinsky) indicated that high-level expression of MAOA was strongly associated with advanced Gleason score ( 8 to 10) (Figure 10B, left panels in both rows). We further correlated MAOA expression profiling with other clinical indices indicating poor prognosis of $\mathrm{PCa}$, including metastasis (lymph node, bone, and soft tissues), seminal vesicle invasion, and biochemical recurrence. MAOA expression was increased when cancer cells formed metastatic lesions after dissemination from the primary site (Figure 10B, top middle panel). Invasion of the muscular wall of the seminal vesicles by PCa is considered as another marker for poor prognosis, metastatic disease, and quick biochemical recurrence (41). Examining the Glinsky data set, we found that PCa patients with seminal vesicle invasion demonstrated higher MAOA expression than patients with intact seminal vesicles (Figure 10B, bottom middle panel). Additionally, analysis of 2 data sets (Taylor 3 and Glinsky) revealed increased MAOA expression in PCa patients who had biochemical recurrence at 5 years (Figure 10B, right panels in both rows). Overall, these clinical data support the experimentally described functional roles of MAOA in PCa and further indicate its prognostic value for distinguishing aggressive from indolent $\mathrm{PCa}$.

In summary, our data suggest that the increased intrinsic MAOA in high-Gleason grade PCa activates a mesenchymal transition and consequent invasive behavior by a mechanism that involves its ability to stabilize HIF $1 \alpha$ via ROS production and activate the VEGF-A/ NRP1-mediated signaling network, which drives EMT by activating AKT/FOXO1 signaling and enhancing nuclear TWIST1 expression via sequestration of FOXO1 in the cytoplasm (Figure 11). Our data further show the key features of this enhanced cell signaling network in prostate tumor xenograft samples and in clinical highGleason grade PCa specimens, supporting the essential roles of $\mathrm{MAOA}$ in prostate tumorigenesis and cancer metastasis.

\section{Discussion}

Our study explores extensively how MAOA affects PCa growth and metastasis and demonstrates, for the first time to our knowledge, that MAOA induces EMT and augments hypoxic responses to increase the migratory, invasive, and metastatic potential of PCa cells. Increased MAOA expression has been previously reported in several types of cancer, including $\mathrm{PCa}(7)$ and renal cell carcinoma (42). Downregulation of MAOA has also been linked with the majority of other cancer types according to a preliminary analysis of an ensemble of cancer data sets (43). The fact that MAOA is expressed differentially in various cancers suggests that MAOA may be regulated differently in the context of specific cancer types. By examining what signaling events may upregulate MAOA in PCa specifically, we demonstrated that select oncogenic events (c-Myc overexpression and the loss of PTEN and p53) that appear to show high frequency in $\mathrm{PCa}(44-46)$ as well as activated androgen signaling were able to induce MAOA in PCa (Supplemental Figure 16). These regulations, particularly the induction of androgen by MAOA, may be an important underlying clue for the expression and function of MAOA in PCa distinct from other types of cancer. Moreover, serotonin, a monoamine neurotransmitter degraded preferentially by MAOA, has been shown to act as a growth factor for several types of cancer $(47,48)$, including melanoma (49) and cholangiocarcinoma (50). Inhibition of MAOA by clorgyline, which elevated serotonin levels, showed protection of melanoma cells against cell death (51). In addition, a suppression of MAOA expression that correlates with worse clinical prognosis and survival was associated with abnormally high levels of seroto- 
nin in cholangiocarcinoma, which stimulate cholangiocarcinoma cell growth, and this condition is responsive to the blockade by the inhibition of serotonin synthesis $(50,52)$. In contrast, serotonin by itself plays a marginal role in regulating the growth of PCa cells in general (53), and there was no significant clinical correlation of serotonin levels with $\mathrm{PCa}$ disease progression $(54,55)$. The different effects of serotonin in diverse cancers therefore also provide insights into the differential functions of MAOA in PCa and other cancers. In addition, given the differential expressions of MAOA in multiple cancers, it would be worthwhile to examine MAOA effects on EMT and hypoxia in other types of cancer to gain a more comprehensive in-depth understanding of how MAOA functions in cancer generally.

MAOA was shown to stabilize HIF1 $\alpha$, activate the VEGF-A/ NRP1 system, and induce the expression of TWIST1, an EMT master transcription factor commonly associated with EMT promotion. These signaling components downstream from MAOA were shown to be clinically relevant, as revealed by the differential expressions of these genes in PCa specimens of different Gleason grades. The clinical relevance of EMT has been shown during tumor progression, and certain typical characteristics, including poor differentiation, correlated with aggressive and invasive behavior in high-Gleason grade PCa can result from EMT and EMT-like processes (56). We observed both increased MAOA expression and EMT in the same specimens of high-grade PCa, which, in line with our other results showing that MAOA can drive EMT (Figure 1), provides a molecular basis for the acquisition of a more aggressive phenotype in high-Gleason grade PCa. Our observation is consistent with previous studies showing that pharmacological inhibition of MAOA in PCa cells kept basal prostatic epithelial cells from differentiating into matured glandular structures by reorganizing cell structures and decreasing the expression of basal cytokeratins (9). In addition, a recent clinical survey assessing EMT marker levels in clinical samples with organ-confined PCa revealed that vimentin and TWIST1, among 13 other EMT markers, showed the most promising predictive potential for poor prognosis including biochemical recurrence (57). Our study mechanistically documented the induction of these 2 EMT markers, along with EMT promotion, by MAOA, and higher MAOA expression correlated with poor clinical outcomes in PCa patients, again suggesting its potential prognostic value (Figure 10).

Elevated ROS levels in MAOA-overexpressing cells contributed to increased HIF1 $\alpha$ stabilization and activity (Figures 2 and 3 and Supplemental Figure 8). Conversely, knockdown of MAOA in both cancer cells and xenograft tumors, resulting in reduced ROS levels, decreased HIF $1 \alpha /$ VEGF-A expression and exerted less hypoxic effects (Figures 2 and 6 and Supplemental Figure 8), which was further recapitulated by pharmacological inhibition of MAOA enzymatic activity (Figure 7). Several groups have reported the capability of both endogenous and added ROS to either transcriptionally (58) or posttranslationally (15) regulate HIF1 $\alpha$ activity. We demonstrated that MAOA-produced ROS could modulate HIF1 $\alpha$ activity by suppressing PHD activity, without changes in HIF1A transcription (Figures 2 and 3). MAOA, when catalyzing oxidative reactions in the outer membrane of mitochondria, immediately produces hydrogen peroxide as a by-product that can be further converted into other species of ROS (3). In the present system, we were able to pinpoint the intracellular hydrogen peroxide released specifically from intact mitochondria as well as extracellular general ROS regulated by MAOA in PCa cells (Figure 6 and Supple- mental Figure 8). The secreted ROS in the extracellular environment may largely serve as paracrine stimuli to further enhance HIF1 $\alpha$ activity via PHD repression in a heterogeneous tumor cell population. Subsequently, the increased HIF1 $\alpha$ expression has the potential to further induce mitochondrial activity including the formation of specific ROS during hypoxia $(19,59)$, potentially programming a "vicious cycle" or feed-forward loop among MAOA, ROS, and HIF1 $\alpha$ to further drive PCa tumorigenesis. The vigorous characterization of the role of MAOA-regulated ROS solely contributed by enzymatic reactions led us further to investigate whether MAOA enzymatic activity or the protein is the determinant for mediating MAOA function in PCa. By conducting a comparative study using WT and a mutated MAOA expression construct that is defective in MAOA enzymatic activity but maintains intact protein expression (60), we demonstrated that MAOA enzymatic activity rather than the protein is the major player driving the migration, invasion, proliferation, and colonization of $\mathrm{PCa}$ cells by enhanced EMT and hypoxia (Supplemental Figure 17), which reinforces the role of MAOA-regulated ROS as an important mediator for MAOA functions in PCa.

The ability of MAOA to control VEGF-A/NRP1 signaling by upregulating the expression of both establishes a connection between this system and downstream signaling pathways. Since the seminal observation that NRP1 can function as a VEGF coreceptor (21), subsequent studies have demonstrated its functional importance in angiogenesis and cancer development (61). MAOA upregulates both the ligand and receptor and mediates receptor function mainly through the angiogenic aspect, which activates the VEGF-A/NRP1 system, particularly its EMT-enhancing ability (Figure 4). These findings support the potential role of MAOA in PCa tissues with high Gleason grade, distinguished by high tumor angiogenesis activity from low-grade PCa. In addition to interactions with angiogenic factors, NRP1 also cooperates with other growth factor receptors, such as c-Met $(62,63)$ and TGF- $\beta$ receptors $(64,65)$, to mediate relevant pathways that contribute to tumorigenesis, and therefore could serve as a prognostic marker as well as an attractive target for cancer therapy. This establishes an additional rationale for targeting MAOA in PCa therapy, since patients with high-Gleason grade PCa may benefit from the blockade of VEGF-A/NRP1-mediated tumor angiogenesis along with other neuropilin-dependent pathways.

One of the salient features of our study is the discovery that MAOA represses E-cadherin transcription and promotes EMT in PCa cells by activating the transcription of TWIST1 via AKT/ FOXO1 signaling. We found that MAOA regulated TWIST1 most robustly among the several master EMT transcription factors we screened. Given the prominent effect of TWIST1 on the repression of the epithelial phenotype by direct suppression of E-cadherin transcription (66), the MAOA/TWIST1 axis can thus be considered an effective regulatory node for the downregulation of E-cadherin by MAOA (Figure 1). The activation of TWIST1 by MAOA is associated with the regulation of FOXO1 activity by AKT signaling, a transcription factor important for cell death and survival. The direct transcriptional regulation of TWIST1 by FOXO1, proposed here for the first time to our knowledge, involves a key response element within the TWIST1 promoter, which is highly conserved in the amniote genomes examined. This finding is supported by our mutational analysis of this element in the TWIST1 promoter as well as the observation that FOXO1 can be recruited to this site in vivo (Figure 5). Moreover, we also 
took into account other possible mechanisms that may contribute to TWIST1 activation in the MAOA context, such as HIF1 $\alpha$ activation of TWIST1 by binding directly to a hypoxia-response element in the TWIST1 promoter as described in a recent study (67). We showed that MAOA was capable of inducing TWIST1 expression in response to effective knockdown of HIF1 $\alpha$, suggesting diverse regulations of TWIST1 in the MAOA context (Supplemental Figure 18). This result further supports our mechanistic finding on the regulation of TWIST1 by MAOA/AKT/FOXO1 signaling in a HIF1 $\alpha$-independent manner. Despite their all being a consequence of the activation of VEGF-A/NRP1 and AKT signaling to drive EMT, the mechanisms we elucidated for the regulation of TWIST1 by MAOA are distinct from those exerted by other genes, such as ER $\beta$, which is engaged in the activation of GSK-3 $\beta$ and SNAIL1 to promote EMT (68). In our studies, we did not detect ER $\beta$ changes in the context of MAOA overexpression (Supplemental Figure 19), suggesting that MAOA's effects in PCa are independent of ER $\beta$ signaling. Notably, the complex but select activation of different pathways for EMT initiation suggests a context-dependent regulation of EMT. In addition to the profound effect on promoting and maintaining EMT (25), TWIST1 has also been implicated in the regulation of cell growth. Recent evidence has indicated that persistent TWIST1 expression induced growth arrest in EMT-like breast cancer cells mimicked by transient TGF- $\beta$ treatment, which was mechanistically associated with cooperation with SNAIL1 and a signal feedback loop involving p38 and extracellular signal-regulated kinase (ERK) (69). By contrast, we observed an opposite effect of TWIST1 on cell proliferation (Supplemental Figure 12C), which, however, is consistent with the findings reported by several other groups where genetic interference with TWIST1 expression decreased PCa cell proliferation independent of cellular androgen levels and responses $(70,71)$. Furthermore, we did not find any changes in SNAIL1 level and p38 and ERK1/2 activity in the context of MAOA (Supplemental Figure 20), which could be an underlying mechanism accounting for the TWIST1 effect in the present system. These controversial results suggest, once again, a potential cell context-dependent role for TWIST1 in cell growth, related possibly to cell types, relevant gene expression profiling, and the responses of cancer cells to environmental cues.

Our study shows that genetic intervention with MAOA expression, by avoiding the potential off-target effects of pharmacological inhibitors, significantly impeded PCa progression or even eliminated prostate tumorigenesis in mice, using tumor xenograft models established from multiple human and murine prostate carcinoma cell lines (Figure 6). Moreover, inhibition of MAOA enzymatic activity by the small-molecule clorgyline achieved growth-inhibitory effects on xenograft tumors (Figure 7 ). The mechanisms discussed here suggest that targeting MAOA blocks PCa tumor growth by disrupting or disengaging the convergent signaling network involving EMT, hypoxia, and oxidative stress (Figure 11). In addition, genetic silencing of MAOA abolished the metastasis of an aggressive androgen-independent $\mathrm{PCa}$ cell line in mice (Figure 8), reinforcing the critical roles of MAOA in EMT initiation and cell invasiveness.

In summary, we have uncovered the underlying molecular mechanisms contributing to MAOA-initiated PCa progression. MAOA was shown to induce EMT, stabilize HIF1 $\alpha$, and mediate hypoxia-elicited elevation of ROS in prostate carcinomas. Elevated MAOA signaling also increased the expression of VEGF-A and its coreceptor NRP1, which together enhance EMT by stimulating AKT/FOXO1 signaling and promoting TWIST1 expression through sequestration of FOXO1 in the cytoplasm. The molecular basis of MAOA action could serve as a prognostic biomarker for poor differentiation and increased aggressiveness in PCa. Targeting MAOA and disengaging its downstream signaling network driving EMT, hypoxia, and oxidative stress provides a promising mechanistic rationale for therapeutic development.

\section{Methods}

Clinical specimens. All tissue specimens used in this study were archived formalin-fixed paraffin-embedded (FFPE) PCa tissues. These FFPE primary PCa specimens of defined Gleason grades were obtained from the Department of Pathology, Xijing Hospital, Fourth Military Medical University (FMMU). PCa tissue microarrays, including a total of 74 primary adenocarcinomas with patient overall survival data, were obtained from Imgenex and US Biomax. Specimens were stained with antibodies specific for MAOA (Santa Cruz), E-cadherin (Cell Signaling), vimentin (Santa Cruz), HIF1 $\alpha$ (Novus Biologicals), VEGF-A (Santa Cruz), FOXO1 (Millipore), pFOXO1 (Millipore), and TWIST1 (Sigma-Aldrich) following our published protocol $(72,73)$ with minor modifications as described below. Additional details on the clinical specimens used and methods of IHC analysis are provided in the Supplemental Methods online.

Cells and reagents. Human PCa PC- 3 and LNCaP cell lines were obtained from American Type Culture Collection. Human PCa C4-2 (36) and $\operatorname{ARCaP}_{M}(35,39)$ cell lines were established by our laboratory. The murine PCa MPC3 cell line, bearing a double knockout of Pten and p53, was provided by Neil Bhowmick (Cedars-Sinai Medical Center). For hypoxia experiments, cells were grown in a hypoxic chamber $\left(1 \% \mathrm{O}_{2}, 5 \% \mathrm{CO}_{2}\right)$. Human $M A O A$ expression construct was generated by insertion of the human $M A O A$ coding region at $E c o R I-B g l I I$ sites in $3 \times$ FLAG-pCMV vector (Sigma-Aldrich) containing a neomycin-resistant gene. Human E-cadherin promoter luciferase reporter construct (pGL2Basic-EcadK1) was provided by Eric Fearon (University of Michigan, Ann Arbor, MI) and obtained from Addgene. Human constitutively active (AAA) FOXO1 expression construct was provided by Kun-Liang Guan (University of California, San Diego, CA) and obtained from Addgene. Human TWIST1 promoter luciferase reporter constructs of various lengths were provided by Lu-Hai Wang (Mount Sinai Hospital, New York, NY). The Renilla luciferase plasmid was purchased from Promega. Human MAOA, NRP1, and nontargeting control shRNA lentiviral particles were purchased from Sigma-Aldrich or Santa Cruz. NAC and clorgyline were purchased from Sigma-Aldrich. DMOG and MG132 were purchased from Millipore. Additional details on cells and reagents used in this study are provided in the Supplemental Methods.

Biochemical analyses. Total RNA was isolated using the RNeasy Mini Kit (Qiagen) and reverse-transcribed to cDNA by M-MLV reverse transcriptase (Promega) as described previously (74). Details on primers and methods used for qPCR are provided in the Supplemental Methods. For immunoblots, cells were extracted with radioimmunoprecipitation assay buffer in the presence of a protease and phosphatase inhibitor cocktail (Thermo Scientific), and blots were performed as described previously $(75,76)$ using primary antibodies against MAOA (Santa Cruz), E-cadherin (Cell Signaling), vimentin (Santa Cruz), N-cadherin (Santa Cruz), TWIST1 (Santa Cruz), $\beta$-actin (Sigma-Aldrich), HIF1 $\alpha$ (BD Biosciences), hydroxy-HIF1 $\alpha$ (Cell Signaling), PHD1-4 (Novus Biologicals), NRP1 (Santa Cruz), phospho-AKT (Ser473) (Cell Signaling), AKT (Cell Signaling), phospho-FOXO1 (Thr24) (Cell Signaling), FOXO1 (Santa Cruz), lamin B1 (Cell Signaling), or GAPDH (Cell Signaling). Immunoblots were further subjected to morphometric and statistical analysis by ImageJ software (NIH) (Supplemental Figure 21). Nuclear/cytoplasmic and mitochondrial/cytoplasmic extracts used 
for immunoblots were prepared with individual kits (Thermo Scientific). VEGF-A levels in culture media were quantified by ELISA (R\&D Systems).

Migration and invasion assays. Assays were performed using $6.5-\mathrm{mm}$ transwell inserts (8- $\mu \mathrm{m}$ pore size) coated with either collagen I or Growth Factor Reduced Matrigel (BD Biosciences) for the migration and invasion assays, respectively. Cells were serum-starved overnight before seeding to eliminate the interference of proliferative effect with cell migration or invasion. Cells were seeded inside transwell inserts containing culture medium without serum. After 18-24 hours, the cells that translocated to the lower surface of the filters were fixed in $4 \%$ formaldehyde. The fixed membranes were stained using $1 \%$ crystal violet. Assays were quantified by counting of the number of stained nuclei in 5 independent fields in each transwell.

Luciferase assays. PC-3 cells were transfected with the desired Firefly luciferase reporter plasmids and the Renilla luciferase construct to normalize for transfection efficiency. Relative light units were calculated as the ratio of Firefly luciferase to Renilla luciferase activity. The protocol used for transfection and measurement of luciferase activity has been described previously (77).

ROS measurement. Cellular ROS was measured according to published protocols $(78,79)$. Briefly, cells were washed with PBS and incubated with $5 \mu \mathrm{M}$ CM- $\mathrm{H}_{2}$ DCFDA (Life Technologies) for 30 minutes. Cells were trypsinized, and mean FL1 fluorescence was measured by flow cytometry. Intact cellular or tumor mitochondria were isolated with a kit (Thermo Scientific) or according to published protocols (80), respectively, and the hydrogen peroxide generation rate was measured for a period of $30 \mathrm{~min}$ utes using the Amplex Red Hydrogen Peroxide Assay Kit (Life Technologies) by a spectrophotometer.

Analyses of Twist 1 promoter. Serial deletion analysis of TWIST1 promoter was used to locate the putative FOXO1-response element. Site-directed mutagenesis was used to mutate the DAF-16 binding element (DBE) sequence from CCAAACT to CCGCGCT. ChIP analysis was used to determine the direct association of endogenous FOXO1 protein with the native TWIST1 promoter in control and MAOA-overexpressing PC-3 cells. Details of these analyses are provided in Supplemental Methods.

Animal studies. Male 4- to 6-week-old athymic nude mice, SCID-beige mice, and immune-intact C57BL/6 mice were purchased from Taconic, housed in the animal research facility at Cedars-Sinai Medical Center (CSMC), and fed a normal chow diet. For xenograft studies, $1 \times 10^{6} \mathrm{LNCaP}, \mathrm{C} 4-2, \mathrm{ARCaP}_{\mathrm{M}}$ (shCon and shMAOA), or PC-3 (vector and MAOA-overexpression) cells were mixed 1:1 with Matrigel (BD Biosciences) and injected s.c. into nude mice, and $1 \times 10^{6} \mathrm{MPC} 3$ (shCon and shMAOA) cells were injected s.c. into immune-intact C57BL/ 6 mice. Each mouse was injected on both flanks. Four to seven mice were used for each group. Details on the number of mice and injection sites used for each cell line to establish subcutaneous tumor xenografts are provided in Supplemental Table 1. Tumor size was measured every 2-3 days by caliper from the time of the formation of palpable tumors, and tumors were dissected and weighed after 3-6 weeks. Tumor volume was calculated by the formula of length $\times$ width ${ }^{2} \times 0.52$ (81). Tumors were fixed in $4 \%$ formaldehyde and embedded in paraffin. Sections were stained with $\mathrm{H} \& \mathrm{E}$ in accordance with standard procedures (82).

For determining the effect of clorgyline on tumor growth, $1 \times 10^{6} \mathrm{C} 4-2$ cells were mixed 1:1 with Matrigel and injected s.c. into nude mice, with each mouse bearing 1 tumor on the right flank. Mice were randomly assigned to 2 groups (clorgyline treatment vs. control, $n=5$ for each) when the tumors reached a measurable size of $100-200 \mathrm{~mm}^{3}$. Daily i.p. injection of clorgyline $(10 \mathrm{mg} / \mathrm{kg})$ was given to mice of the treatment group for 21 days, and saline injection was used for the control group. Tumor size was measured every 3 days by caliper, and tumors were weighed when mice were subjected to necropsy on day 21 .
For metastatic studies, $1 \times 10^{6} \mathrm{ARCaP}_{\mathrm{M}}$ cells tagged with a Firefly luciferase reporter construct (Promega) were injected into the left cardiac ventricle of anesthetized SCID-beige mice as described previously (39). Development of metastases was monitored by BLI. Anesthetized mice were injected i.p. with $30 \mathrm{mg}$ D-Luciferin per mouse (Gold Biotech). Bioluminescence images were acquired with a Xenogen IVIS Spectrum Imaging System (PerkinElmer). Analysis was performed with Living Image software (PerkinElmer) by measurement of photon flux in the whole bodies of mice. Data were normalized to the signal in week 1.

Immunohistochemistry. IHC analysis of tumor xenograft or mouse primary tumor samples was performed using antibodies against Ki-67 (Dako), MAOA (Santa Cruz), E-cadherin (Cell Signaling), vimentin (Santa Cruz), HIF1 $\alpha$ (BD Biosciences), or VEGF-A (Santa Cruz) following our published protocol (72) with minor modifications. Briefly, FFPE sections $(4 \mu \mathrm{m})$ were deparaffinized, rehydrated, and subjected to antigen retrieval. After incubation in Dual Endogenous Enzyme Block solution (Dako) for 10 minutes, the section was treated with primary antibody diluted by different folds with Antibody Diluent solution (Dako) at $4{ }^{\circ} \mathrm{C}$ overnight. The section was then washed 3 times in PBST (PBS containing 0.2\% Tween-20) for 5 minutes per washing. To detect specific staining, the section was treated for 30 minutes with EnVision + Dual Link System-HRP (Dako), which contained HRP-conjugated goat antibodies against mouse and rabbit IgG. The section was washed 3 times for 5 minutes each, and specific stains were developed with 3'3-diaminobenzidine (Dako). Image acquisition was performed using a Nikon camera and software. Magnification was $\times 400$ (scale bars $\sim 20 \mu \mathrm{m}$ ).

MAOA enzymatic activity assay. MAOA enzymatic activity was determined in control and MAOA-knockdown cells and tumor samples as described previously (83). Briefly, $100 \mu \mathrm{g}$ of total protein was incubated with $1 \mathrm{mM}$ $\left[{ }^{14} \mathrm{C}\right] 5$-HT in the assay buffer at $37^{\circ} \mathrm{C}$ for 20 minutes, and the reaction was terminated by the addition of ice-cold $6 \mathrm{~N} \mathrm{HCl}$. The reaction products were extracted with benzene/ethyl acetate $(1: 1)$ and centrifuged at $4^{\circ} \mathrm{C}$ for $7 \mathrm{~min}$ utes. The organic phase containing the reaction products was extracted, and radioactivity was determined by liquid scintillation spectroscopy.

Microarray data sets. Two PCa DNA microarray data sets, Taylor 3 (84) and Glinsky (85), were downloaded directly from the Oncomine database by licensed access. Microarray data of the Taylor 3 data set are also publicly available in Gene Expression Omnibus (GEO GSE21032).

Statistics. Data are presented as the mean \pm SEM as indicated in the figure legends. Comparisons between Kaplan-Meier curves were performed using the log-rank test. All other comparisons were analyzed by unpaired 2-tailed Student's $t$ test. A $P$ value less than 0.05 was considered statistically significant.

Study approval. All animal studies received prior approval by the IACUC of CSMC and were conducted in compliance with its recommendations. All human studies were reviewed and approved by the IRB of CSMC and FMMU, and written informed consent was provided for human samples.

\section{Acknowledgments}

This work was supported by NIH/National Cancer Institute grants 5P01CA098912 and R01CA122602, the Board of Governors Cancer Research Chair, and the Steven Spielberg Fund in Prostate Cancer Research (to L.W.K. Chung); Department of Defense Prostate Cancer Research Program grant W81XWH-12-1-0282 (to H.E. Zhau and J.C. Shih); and the Daniel Tsai Family Fund (to J.C. Shih). We thank Lei Zhang (FMMU, Xi'an, Shaanxi, China) for providing clinical specimens, Pradip Roy-Burman (Department of Pathology, University of Southern California, Los Angeles, CA) for providing mouse prostate-specific Pten-knockout tumor specimens, and Bin Qian and Jenny Wu (Department of Pharmacology and Pharmaceuticals, University of Southern California, Los 
Angeles, CA) for providing technical assistance. We also thank Gary Mawyer for editorial assistance.

Received for publication May 20, 2013, and accepted in revised form April 3, 2014.

Address correspondence to: Leland W.K. Chung, Cedars-Sinai Medical Center, 8750 Beverly Boulevard, Atrium 103, Los Angeles,
California 90048, USA. Phone: 310.423.7622; Fax: 310.423.8543; E-mail: leland.chung@cshs.org. Or to: Jean C. Shih, University of Southern California, 1985 Zonal Avenue, PSC 518, Los Angeles, California 90089, USA. Phone: 323.442.1441; Fax: 323.442.3229; E-mail: jcshih@usc.edu. Or to: Haiyen E. Zhau, Cedars-Sinai Medical Center, 8750 Beverly Boulevard, Atrium 103, Los Angeles, California 90048, USA. Phone: 310.423.8179; Fax: 310.423.8543; E-mail: haiyen.zhau@cshs.org.
1. Siegel R, Naishadham D, Jemal A. Cancer statistics, 2013. CA Cancer J Clin. 2013;63(1):11-30.

2. Partin AW, et al. Combination of prostate-specific antigen, clinical stage, and Gleason score to predict pathological stage of localized prostate cancer. A multi-institutional update. JAMA. 1997; 277(18):1445-1451.

3. Shih JC, Chen K, Ridd MJ. Monoamine oxidase: from genes to behavior. Annu Rev Neurosci. 1999; 22:197-217.

4. Shih JC, Wu JB, Chen K. Transcriptional regulation and multiple functions of MAO genes. J Neural Transm. 2011;118(7):979-986.

5. Bortolato M, Chen K, Shih JC. Monoamine oxidase inactivation: from pathophysiology to therapeutics. Adv Drug Deliv Rev. 2008;60(13-14):1527-1533.

6. Trachootham D, Alexandre J, Huang P. Targeting cancer cells by ROS-mediated mechanisms: a radical therapeutic approach? Nat Rev Drug Discov. 2009;8(7):579-591.

7. True L, et al. A molecular correlate to the Gleason grading system for prostate adenocarcinoma. Proc Natl Acad Sci U S A. 2006;103(29):10991-10996.

8. Peehl DM, Coram M, Khine H, Reese S, Nolley R, Zhao $\mathrm{H}$. The significance of monoamine oxidase-A expression in high grade prostate cancer. J Urol. 2008;180(5):2206-2211.

9. Zhao H, Nolley R, Chen Z, Reese SW, Peehl DM. Inhibition of monoamine oxidase A promotes secretory differentiation in basal prostatic epithelial cells. Differentiation. 2008;76(7):820-830.

10. Nauseef JT, Henry MD. Epithelial-to-mesenchymal transition in prostate cancer: paradigm or puzzle? Nat Rev Urol. 2011;8(8):428-439.

11. Stewart GD, Ross JA, McLaren DB, Parker CC, Habib FK, Riddick AC. The relevance of a hypoxic tumour microenvironment in prostate cancer. BJU Int. 2010;105(1):8-13.

12. Gupta-Elera G, Garrett AR, Robison RA, O'Neill $\mathrm{KL}$. The role of oxidative stress in prostate cancer. Eur J Cancer Prev. 2012;21(2):155-162.

13. Gleason DF, Mellinger GT. Prediction of prognosis for prostatic adenocarcinoma by combined histological grading and clinical staging. J Urol. 1974;111(1):58-64.

14. Lu X, Kang Y. Hypoxia and hypoxia-inducible factors: master regulators of metastasis. Clin Cancer Res. 2010;16(24):5928-5935.

15. Finley LW, et al. SIRT3 opposes reprogramming of cancer cell metabolism through HIF1 $\alpha$ destabilization. Cancer Cell. 2011;19(3):416-428.

16. Kaelin WG Jr, Ratcliffe PJ. Oxygen sensing by metazoans: the central role of the HIF hydroxylase pathway. Mol Cell. 2008;30(4):393-402.

17. Schnaitman C, Erwin VG, Greenawalt JW. The submitochondrial localization of monoamine oxidase. An enzymatic marker for the outer membrane of rat liver mitochondria. J Cell Biol. 1967;32(3):719-735.

18. Wang GL, Jiang BH, Rue EA, Semenza GL. Hypoxiainducible factor 1 is a basic-helix-loop-helix-PAS heterodimer regulated by cellular $\mathrm{O} 2$ tension. Proc Natl Acad Sci U S A. 1995;92(12):5510-5514.

19. Chandel NS, Maltepe E, Goldwasser E, Mathieu CE, Simon MC, Schumacker PT. Mitochondrial reactive oxygen species trigger hypoxia-induced transcription. Proc Natl Acad Sci US A. 1998;95(20):11715-11720.

20. Antonarakis ES, Carducci MA. Targeting angio- genesis for the treatment of prostate cancer. Expert Opin Ther Targets. 2012;16(4):365-376.

21. Soker S, Takashima S, Miao HQ, Neufeld G, Klagsbrun M. Neuropilin-1 is expressed by endothelial tumor cells as an isoform-specific receptor for vascular endothelial growth factor. Cell. 1998;92(6):735-745.

22. Bartholomeusz C, Gonzalez-Angulo AM. Targeting the PI3K signaling pathway in cancer therapy Expert Opin Ther Targets. 2012;16(1):121-130.

23. Zhang Y, Gan B, Liu D, Paik JH. FoxO family members in cancer. Cancer Biol Ther. 2011;12(4):253-259.

24. Bachelder RE, et al. Vascular endothelial growth factor is an autocrine survival factor for neuropilin-expressing breast carcinoma cells. Cancer Res. 2001;61(15):5736-5740.

25 . Yang J, et al. Twist, a master regulator of morphogenesis, plays an essential role in tumor metastasis. Cell. 2004;117(7):927-939.

26. Wallerand $\mathrm{H}$, et al. The epithelial-mesenchymal transition-inducing factor TWIST is an attractive target in advanced and/or metastatic bladder and prostate cancers. Urol Oncol. 2010;28(5):473-479.

27. Vichalkovski A, Gresko E, Hess D, Restuccia DF, Hemmings BA. PKB/AKT phosphorylation of the transcription factor Twist- 1 at Ser42 inhibits p53 activity in response to DNA damage. Oncogene. 2010;29(24):3554-3565.

28. Xue G, et al. Akt/PKB-mediated phosphorylation of Twist 1 promotes tumor metastasis via mediating cross-talk between PI3K/Akt TGF-beta signaling axes. Cancer Discov. 2012;2(3):248-259.

29. Shiota M, et al. Foxo3a suppression of urothelial cancer invasiveness through Twist1, Y-box-binding protein 1, E-cadherin regulation. Clin Cancer Res. 2010;16(23):5654-5663

30. Tang ED, Nunez G, Barr FG, Guan KL. Negative regulation of the forkhead transcription factor FKHR by Akt. J Biol Chem. 1999;274(24):16741-16746.

31. Cheng GZ, et al. Twist is transcriptionally induced by activation of STAT3 and mediates STAT3 oncogenic function. J Biol Chem. 2008;283(21):14665-14673.

32. Glauser DA, Schlegel W. The emerging role of FOXO transcription factors in pancreatic $\beta$ cells. $J$ Endocrinol. 2007;193(2):195-207.

33. Allen DL, Unterman TG. Regulation of myostatin expression and myoblast differentiation by FoxO and SMAD transcription factors. Am J Physiol Cell Physiol. 2007;292(1):C188-C199.

34. Fan W, et al. FoxO1 regulates Tlr4 inflammatory pathway signalling in macrophages. EMBO J. 2010;29(24):4223-4236.

35. Zhau HY, et al. Androgen-repressed phenotype in human prostate cancer. Proc Natl Acad SciUS A. 1996; 93(26):15152-15157.

36. Thalmann GN, et al. Androgen-independent cancer progression and bone metastasis in the $\mathrm{LNCaP}$ model of human prostate cancer. Cancer Res. 1994;54(10):2577-2581.

37. Green AR, Youdim MB. Effects of monoamine oxidase inhibition by clorgyline, deprenil or tranylcypromine on 5-hydroxytryptamine concentrations in rat brain hyperactivity following subsequent tryptophan administration. BrJ Pharmacol. 1975;55(3):415-422.

38. Wu TT, et al. Establishing human prostate cancer cell xenografts in bone: induction of osteoblastic reaction by prostate-specific antigen-produc- ing tumors in athymic and SCID/bg mice using LNCaP and lineage-derived metastatic sublines. Int J Cancer. 1998;77(6):887-894.

39. Xu J, et al. Prostate cancer metastasis: role of the host microenvironment in promoting epithelial to mesenchymal transition and increased bone and adrenal gland metastasis. Prostate. 2006;66(15):1664-1673.

40. Tomlins SA, et al. Recurrent fusion of TMPRSS2 and ETS transcription factor genes in prostate cancer. Science. 2005;310(5748):644-648.

41. Potter SR, Epstein JI, Partin AW. Seminal vesicle invasion by prostate cancer: prognostic significance and therapeutic implications. Rev Urol. 2000; 2(3):190-195.

42. Hodorova I, et al. Comparison of expression pattern of monoamine oxidase A with histopathologic subtypes and tumour grade of renal cell carcinoma. Med Sci Monit. 2012;18(12):BR482-BR486.

43. Rybaczyk LA, Bashaw MJ, Pathak DR, Huang K. An indicator of cancer: downregulation of monoamine oxidase-A in multiple organs and species. BMC Genomics. 2008;9:134.

44. Gurel B, et al. Nuclear MYC protein overexpression is an early alteration in human prostate carcinogenesis. Mod Pathol. 2008;21(9):1156-1167.

45. McMenamin ME, Soung P, Perera S, Kaplan I, Loda M, Sellers WR. Loss of PTEN expression in paraffin-embedded primary prostate cancer correlates with high Gleason score and advanced stage. Cancer Res. 1999;59(17):4291-4296.

46. Chi SG, deVere White RW, Meyers FJ, Siders DB, Lee F, Gumerlock PH. p53 in prostate cancer: frequent expressed transition mutations. J Natl Cancer Inst. 1994;86(12):926-933.

47. Pratesi G, Cervi S, Balsari A, Bondiolotti G, Vicentini LM. Effect of serotonin and nicotine on the growth of a human small cell lung cancer xenograft. Anticancer Res. 1996;16(6B):3615-3619.

48. Soll C, et al. Serotonin promotes tumor growth in human hepatocellular cancer. Hepatology. 2010; 51(4):1244-1254.

49. Lee HJ, Park MK, Kim SY, Park Choo HY, Lee AY, Lee $\mathrm{CH}$. Serotonin induces melanogenesis via serotonin receptor 2A. BrJ Dermatol. 2011;165(6):1344-1138.

50. Alpini $\mathrm{G}$, et al. Serotonin metabolism is dysregulated in cholangiocarcinoma, which has implications for tumor growth. Cancer Res. 2008;68(22):9184-9193.

51. Malorni W, et al. Protection against apoptosis by monoamine oxidase A inhibitors. FEBS Lett. 1998; 426(1):155-159.

52. Huang L, et al. Monoamine oxidase A expression is suppressed in human cholangiocarcinoma via coordinated epigenetic and IL-6-driven events. Lab Invest. 2012;92(10):1451-1460.

53. Pirozhok I, Meye A, Hakenberg OW, Fuessel S, Wirth MP. Serotonin and melatonin do not play a prominent role in the growth of prostate cancer cell lines. Urol Int. 2010;84(4):452-460.

54. Jungwirth N, Haeberle L, Schrott KM, Wullich B, Krause FS. Serotonin used as prognostic marker of urological tumors. World J Urol. 2008;26(5):499-504.

55. Heinrich E, et al. Neuroendocrine tumor cells in prostate cancer: evaluation of the neurosecretory products serotonin, bombesin, and gastrin - impact on angiogenesis and clinical follow-up. Prostate. 2011;71(16):1752-1758.

56. Savagner P. The epithelial-mesenchymal tran- 
sition (EMT) phenomenon. Ann Oncol. 2010; 21(suppl 7):vii89-vii92

57. Behnsawy HM, Miyake H, Harada K, Fujisawa M. Expression patterns of epithelial-mesenchymal transition markers in localized prostate cancer: significance in clinicopathological outcomes following radical prostatectomy. BJU Int. 2013;111(1):30-37.

58 . Bonello $S$, et al. Reactive oxygen species activate the HIF-1 $\alpha$ promoter via a functional NFKB site. Arterioscler Thromb Vasc Biol. 2007;27(4):755-761.

59. Chandel NS, et al. Reactive oxygen species generated at mitochondrial complex III stabilize hypoxiainducible factor- $1 \alpha$ during hypoxia: a mechanism of O2 sensing. J Biol Chem. 2000;275(33):25130-25138.

60. Geha RM, Chen K, Wouters J, Ooms F, Shih JC. Anal$y$ sis of conserved active site residues in monoamine oxidase $\mathrm{A}$ and $\mathrm{B}$ and their three-dimensional molecular modeling. J Biol Chem. 2002;277(19):17209-17216.

61. Guttmann-Raviv N, Kessler O, Shraga-Heled N, Lange T, Herzog Y, Neufeld G. The neuropilins and their role in tumorigenesis and tumor progression. Cancer Lett. 2006;231(1):1-11.

62. Matsushita A, Gotze T, Korc M. Hepatocyte growth factor-mediated cell invasion in pancreatic cancer cells is dependent on neuropilin-1. Cancer Res. 2007; 67(21):10309-10316.

63. Hu B, et al. Neuropilin-1 promotes human glioma progression through potentiating the activity of the HGF/SF autocrine pathway. Oncogene. 2007; 26(38):5577-5586

64. Glinka Y, Prud'homme GJ. Neuropilin-1 is a receptor for transforming growth factor $\beta$-1, activates its latent form, promotes regulatory $\mathrm{T}$ cell activity. J Leukoc Biol. 2008;84(1):302-310.

65. Cao $S$, et al. Neuropilin-1 promotes cirrhosis of the rodent human liver by enhancing PDGF/TGF- $\beta$ signaling in hepatic stellate cells. J Clin Invest. 2010;
120(7):2379-2394.

66. Vesuna F, van Diest P, Chen JH, Raman V. Twist is a transcriptional repressor of E-cadherin gene expression in breast cancer. Biochem Biophys Res Commun. 2008;367(2):235-241.

67. Yang $\mathrm{MH}$, et al. Direct regulation of TWIST by HIF-1 $\alpha$ promotes metastasis. Nat Cell Biol. 2008; 10(3):295-305.

68. Mak P, et al. ERbeta impedes prostate cancer EMT by destabilizing HIF- $1 \alpha$ and inhibiting VEGF-mediated snail nuclear localization: implications for Gleason grading. Cancer Cell. 2010;17(4):319-332.

69. Tran DD, Corsa CA, Biswas H, Aft RL, Longmore GD. Temporal and spatial cooperation of Snail1 and Twist 1 during epithelial-mesenchymal transition predicts for human breast cancer recurrence. Mol Cancer Res. 2011;9(12):1644-1657.

70. Shiota $\mathrm{M}$, et al. Castration resistance of prostate cancer cells caused by castration-induced oxidative stress through Twist 1 and androgen receptor overexpression. Oncogene. 2010;29(2):237-250.

71. Shiota M, et al. Twist promotes tumor cell growth through YB-1 expression. Cancer Res. 2008; 68(1):98-105

72. Zhau HE, et al. Epithelial to mesenchymal transition (EMT) in human prostate cancer: lessons learned from ARCaP model. Clin Exp Metastasis. 2008; 25(6):601-610.

73. Zeng N, et al. PTEN controls beta-cell regeneration in aged mice by regulating cell cycle inhibitor p16(ink4a.). Aging Cell. 2013;12(6):1000-1011.

74. Wu JB, Chen K, Ou XM, Shih JC. Retinoic acid activates monoamine oxidase $\mathrm{B}$ promoter in human neuronal cells. I Biol Chem. 2009; 284(25):16723-16735.

75. Zeng N, et al. Adaptive basal phosphorylation of eIF2alpha is responsible for resistance to cellular stress-induced cell death in Pten-null hepatocytes. Mol Cancer Res. 2011;9(12):1708-1717.

76. Chen K, Ou XM, Wu JB, Shih JC. Transcription factor E2F-associated phosphoprotein (EAPP), RAM2/CDCA7L/JPO2 (R1), simian virus 40 promoter factor 1 ( $\mathrm{Sp} 1$ ) cooperatively regulate glucocorticoid activation of monoamine oxidase B. Mol Pharmacol. 2011;79(2):308-317.

77. Wu JB, Shih JC. Valproic acid induces monoamine oxidase A via Akt/forkhead box $\mathrm{O} 1$ activation. $\mathrm{Mol}$ Pharmacol. 2011;80(4):714-723.

78. Eruslanov E, Kusmartsev S. Identification of ROS using oxidized DCFDA and flow-cytometry. Methods Mol Biol. 2010;594:57-72.

79. Li Y, et al. Phosphatase and tensin homolog deleted on chromosome 10 (PTEN) signaling regulates mitochondrial biogenesis respiration via estrogen-related receptor $\alpha(E R R \alpha)$. J Biol Chem. 2013; 288(35):25007-25024.

80. Graham JM. Isolation of mitochondria from tissues and cells by differential centrifugation. Curr Protoc Cell Biol. 2001;3:Unit 3.3.

81. Tomayko MM, Reynolds CP. Determination of subcutaneous tumor size in athymic (nude) mice. Cancer Chemother Pharmacol. 1989;24(3):148-154.

82. Fischer AH, Jacobson KA, Rose J, Zeller R. Hematoxylin and eosin staining of tissue and cell sections. CSH Protoc. 2008;2008:pdb.prot4986.

83. Wu JB, Chen K, Li Y, Lau YF, Shih JC. Regulation of monoamine oxidase A by the SRY gene on the Y chromosome. FASEB J. 2009;23(11):4029-4038.

84. Taylor BS, et al. Integrative genomic profiling of human prostate cancer. Cancer Cell. 2010;18(1):11-22.

85. Glinsky GV, Glinskii AB, Stephenson AJ, Hoffman RM, Gerald WL. Gene expression profiling predicts clinical outcome of prostate cancer. J Clin Invest. 2004;113(6):913-923. 